Article

\title{
Sex-Specific Differences in the Relationship between Insulin Resistance and Adiposity Indexes in Children and Adolescents with Obesity
}

\author{
Valeria Calcaterra ${ }^{1,2, *} \mathbb{C}^{\circ}$, Elvira Verduci ${ }^{2,3}$, Laura Schneider ${ }^{2}$, Hellas Cena ${ }^{4,5}{ }^{(0)}$, Annalisa De Silvestri ${ }^{6}{ }^{(}$, \\ Sara Vizzuso ${ }^{2}$, Federica Vinci ${ }^{1,7}$, Chiara Mameli ${ }^{2,8}$ and Gianvincenzo Zuccotti ${ }^{2,8}$ \\ check for \\ updates \\ 1 Pediatrics and Adolescentology Unit, Department of Internal Medicine, University of Pavia, \\ 27100 Pavia, Italy; fede90vinci@gmail.com \\ 2 Department of Pediatrics, "Vittore Buzzi" Children's Hospital, 20154 Milan, Italy; \\ elviraverduci@unimi.it (E.V.); lauraschneider@asst-fbf-sacco.it (L.S.); saravizzuso@asst-fbf-sacco.it (S.V.); \\ chiaramameli@unimi.it (C.M.); gianvincenzozuccotti@unimi.it (G.Z.) \\ 3 Department of Health Sciences, University of Milan, 20146 Milan, Italy \\ 4 Laboratory of Dietetics and Clinical Nutrition, Department of Public Health, Experimental and Forensic \\ Medicine, University of Pavia, 27100 Pavia, Italy; hellas.cena@unipv.it \\ 5 Clinical Nutrition and Dietetics Service, Unit of Internal Medicine and Endocrinology, ICS Maugeri IRCCS, \\ 27100 Pavia, Italy \\ 6 Biometry \& Clinical Epidemiology, Scientific Direction, Fondazione IRCCS Policlinico San Matteo, \\ 27100 Pavia, Italy; a.desilvestri@smatteo.pv.it \\ 7 Pediatric Unit, Fondazione IRCCS Policlinico San Matteo, 27100 Pavia, Italy \\ 8 Department of Biomedical and Clinical Science "L. Sacco", University of Milan, 20157 Milan, Italy \\ * Correspondence: valeria.calcaterra@unipv.it
}

Citation: Calcaterra, V.; Verduci, E.; Schneider, L.; Cena, H.; De Silvestri, A.; Vizzuso, S.; Vinci, F.; Mameli, C.; Zuccotti, G. Sex-Specific Differences in the Relationship between Insulin Resistance and Adiposity Indexes in Children and Adolescents with Obesity. Children 2021, 8, 449. https://doi.org/10.3390/ children 8060449

Academic Editor: Rachana Shah

Received: 22 April 2021

Accepted: 25 May 2021

Published: 26 May 2021

Publisher's Note: MDPI stays neutral with regard to jurisdictional claims in published maps and institutional affiliations.

Copyright: (c) 2021 by the authors. Licensee MDPI, Basel, Switzerland. This article is an open access article distributed under the terms and conditions of the Creative Commons Attribution (CC BY) license (https:/ / creativecommons.org/licenses/by/ $4.0 /)$.
Abstract: New indexes of adiposity have been introduced to evaluate body-fat distribution and cardiometabolic risk. However, data on the correlation between Insulin Resistance (IR) and these new indexes are limited. We therefore evaluated the relationship between IR and adiposity indexes in children and adolescents with obesity, focusing on gender differences. We retrospectively enrolled 586 patients with obesity $(10.80 \pm 2.63 ; 306 \mathrm{~F} / 279 \mathrm{M})$. As adiposity indexes we considered body mass index (BMI), BMI-z score, WC, waist-to-height ratio (WHtR), a body shape index (ABSI), triponderal mass index (TMI), visceral adiposity index (VAI) and conicity index (ConI). The homeostasis model assessment for insulin resistance (HOMA-IR), HOMA of percentage $\beta$-cell function (HOMA- $\beta$ ), quantitative insulin sensitivity check index (QUICKI), and triglyceride and glucose index (TyG-index) were measured and recorded as IR surrogates. In both sexes, WC and VAI significantly correlated with all IR measurements $(p<0.001)$. BMI significantly correlated $(p<0.001)$ with all IR parameters except for the TyG-index in females. Fat mass and TMI correlated with IR parameters only in females, BMI-z score with IR markers except for HOMA- $\beta$ in males, WHtR with HOMA- $\beta$ in both sexes $(p<0.05)$, free fat mass with HOMA-IR and QUICKI only in females $(p<0.01)$, ConI correlated with the TyG index in females $(p=0.01)$. Tryglicerides and SBP were correlated with all IR measurements $(p<0.001)$, in both sexes. Correlations between different sex parameters were significantly more evident in middle puberty. The relationship between IR surrogates and obesity indexes is influenced by gender in pediatrics. Sex-specific differences in obesity-related complications should be considered in preventive intervention decision-making.

Keywords: sex-specific differences; insulin resistance; adiposity; children; adolescents; obesity

\section{Introduction}

Insulin resistance (IR) is a crucial factor contributing to the pathogenetic mechanism of several disorders, including type 2 diabetes mellitus (DMT2), hypertension, dyslipidemia, cardiovascular disease, and metabolic syndrome [1-3]. IR represents the earliest manifestation of dysmetabolism in children [4,5]. 
Euglycemic-hyperinsulinemic clamp is the gold standard method for determining insulin sensitivity. However, it is invasive, time intensive and not practical in the pediatric age $[6,7]$. Consequently, several indirect methods have been proposed for clinical studies and epidemiological evaluations. In particular, surrogate measures, based on fasting levels of insulin and glucose are validated tools that simplify IR measurement and are used in epidemiological studies and in clinical practice [8-10]; these include the homeostasis model assessment for insulin resistance (HOMA-IR), HOMA of percentage $\beta$-cell function (HOMA- $\beta$ ) and quantitative insulin sensitivity check index (QUICKI). The triglyceride and glucose index (TyG-index) may also be useful for IR prediction in large-scale studies or screening of populations at high risk of diabetes, as they are more sensitive in recognizing IR compared with HOMA-IR [11,12].

Body-fat distribution is also a critical player in IR development [13-15]. Body mass index (BMI), waist circumference (WC) and or waist-to-height ratio (WHtR) are usually considered good markers of the dysmetabolic profile [16-22]. Recently, new indexes of obesity, including body shape index (ABSI), triponderal mass index (TMI), conicity index (ConI) and the visceral adiposity index (VAI) have been introduced to evaluate body-fat distribution and cardiometabolic risk [23-32].

Limited data on the correlation between IR and these new indexes of adiposity have been reported in the pediatric age [28,32]. Therefore, we analyzed the association of IR surrogates and adiposity indexes in children and adolescents with obesity. Considering that IR is partly attributed to sex differences in fat mass, we focused the evaluation on sex differences. The relationship between IR and other cardiometabolic risk factors was also considered.

\section{Materials and Methods}

\subsection{Patients}

We retrospectively enrolled 586 Caucasian children and adolescents ( 376 females and 279 males) with obesity (BMI-z score $\geq 2$ according to the World Health Organization), aged $10.80 \pm 2.63$ years (range 6-18 years) referred to the Outpatient Clinics of the Vittore Buzzi Children's Hospital, Milano, Fondazione IRCCS Policlinico S. Matteo, Pavia, and the San Paolo University Hospital of Milan. Patients were referred by their general practitioner or primary care pediatrician between January 2015 and December 2020. Known secondary obesity conditions, use of any medications, and concomitant chronic or acute illnesses were considered exclusion criteria.

\subsection{Physical Examination and Adiposity Indexes}

In all participants, height, weight, pubertal stage, WC, BMI, adiposity indexes, and body composition were measured. Total and HDL-cholesterol levels as well as diastolic (DBP) and systolic blood pressure (SBP) measurements were also collected.

Height, weight, pubertal stage, WC measurement, DBP and SBP were performed as reported elsewhere [12]. As previously reported, pubertal stages were classified as follows, Prepubertal stage $=$ Tanner Stage 1 ; Middle puberty $=$ Tanner Stages 2-3; Late puberty $=$ Tanner Stages 4-5 [12].

BMI was calculated as body weight (kilograms) divided by height (meters squared) and was transformed into BMI-z scores using World Health Organization (WHO) reference values [33].

In addition to BMI, BMI-z score, and WC, adiposity indexes, including WHtR, ABSI, TMI, VAI, ConI were considered and calculated as follows:

- $\quad \mathrm{WHtR}=\mathrm{WC} / \mathrm{Ht}$

- $\mathrm{ABSI}=1000 \times \mathrm{WC} \times \mathrm{Wt}^{-2 / 3} \times \mathrm{Ht}^{5 / 6}[34]$

- $\quad$ TMI = weight $(\mathrm{kg}) /$ height $(\mathrm{m})^{3}[29]$

- $\mathrm{ConI}=\mathrm{WC} /\left(0.109 \times(\mathrm{Wt} / \mathrm{Ht})^{0.5}\right)[35]$

- VAI [36] 


$$
\begin{gathered}
\text { Male }=[\mathrm{WC} /(39.68+(1.88 \times \mathrm{BMI}))] \times(\mathrm{TG} / 1.03) \times(1.31 / \mathrm{HDL}-\mathrm{C}) \\
\text { Female }=[\mathrm{WC} /(36.58+(1.89 \times \mathrm{BMI}))] \times(\mathrm{TG} / 0.81) \times(1.52 / \mathrm{HDL}-\mathrm{C})
\end{gathered}
$$

\subsection{Biochemical Evaluation and Insulin Resistance Measurements}

At enrollment, in addition to the collection of historical data and a physical examination, a biochemical evaluation was performed. Patients underwent a blood draw in a fasting state between 8:30 a.m. and 9:00 a.m. and plasma glucose, insulin, triglycerides (TG), total, and HDL-cholesterol were analyzed the same morning by standard methods.

As IR surrogates we considered the following:

- $\quad$ HOMA-IR calculated as insulin resistance $=($ insulin $\times$ glucose $) / 22.5$ [8];

- $\quad$ TyG-index calculated as $\ln [$ fasting triglycerides $(\mathrm{mg} / \mathrm{dL}) \times$ fasting plasma glucose (mg/dL)/2]) [37];

- HOMA of percentage $\beta$-cell function $($ HOMA- $\beta)$ calculated as HOMA- $\beta \%=($ FIRI $\times$ 20)/(FPG - 3.5) [8];

- Quantitative Insulin sensitivity Check Index (QUICK index) calculated as the inverse of the sum of the logarithms of fasting insulin and fasting glucose: $1 /(\log ($ fasting insulin $\mu \mathrm{U} / \mathrm{mL})+\log ($ fasting glucose $\mathrm{mg} / \mathrm{dL})$ ) [38].

\subsection{Statistical Analysis}

Quantitative variables were described as the mean and standard deviation when normally distributed (Shapiro-Wilks's test). Comparisons based on sex were made with the Student $\mathrm{T}$ test for independent samples. Relationships between the indexes, were assessed with the Pearson correlation coefficient. A $p$-value $<0.05$ was considered statistically significant.

\section{Results}

Anthropometric features, adiposity indexes and indirect IR markers in the patients according to sex, are summarized in Table 1.

Table 1. Indexes of obesity and insulin resistance and metabolic parameters of the patients according

\begin{tabular}{|c|c|c|c|c|}
\hline Parameters & All & Females $(n=306)$ & Males $(n=279)$ & $p^{*}$ \\
\hline Age (years) & $10.80 \pm 2.63$ & $11.4 \pm 3.0$ & $11.2 \pm 2.90$ & 0.98 \\
\hline \multicolumn{5}{|l|}{ Pubertal stages } \\
\hline Tanner 1 & 145 & 89 & 56 & \multirow{3}{*}{$<0.001$} \\
\hline Tanner 2-3 & 375 & 174 & 201 & \\
\hline Tanner 4-5 & 65 & 43 & 22 & \\
\hline Body mass index $\left(\mathrm{kg} / \mathrm{m}^{2}\right)$ & $28.36 \pm 4.40$ & $28.46 \pm 4.84$ & $28.26 \pm 3.87$ & \multirow{4}{*}{0.58} \\
\hline Tanner 1 & $25.13 \pm 3.18 * *$ & $24.88 \pm 2.87 * *$ & $25.54 \pm 3.62 * *$ & \\
\hline Tanner 2-3 & $28.95 \pm 4.15$ & $29.28 \pm 4.72$ & $28.67 \pm 3.60$ & \\
\hline Tanner 4-5 & $32.18 \pm 3.52$ & $32.53 \pm 3.80$ & $31.48 \pm 2.84$ & \\
\hline Body mass index $z$ score & $2.73 \pm 0.73$ & $2.72 \pm 0.72$ & $2.74 \pm 0.74$ & \multirow{4}{*}{0.78} \\
\hline Tanner 1 & $3.07 \pm 1.04^{* *}$ & $3.03 \pm 1.04^{* *}$ & $3.12 \pm 1.04^{* *}$ & \\
\hline Tanner 2-3 & $2.65 \pm 0.57$ & $2.63 \pm 0.50$ & $2.67 \pm 0.62$ & \\
\hline Tanner 4-5 & $2.42 \pm 0.42$ & $2.44 \pm 0.45$ & $2.39 \pm 0.37$ & \\
\hline Waist circumference $(\mathrm{cm})$ & $87.77 \pm 11.28$ & $86.49 \pm 11.22$ & $89.14 \pm 11.18$ & \multirow{4}{*}{0.005} \\
\hline Tanner 1 & $77.62 \pm 7.68^{* *}$ & $77.56 \pm 7.72 * *$ & $77.72 \pm 7.67^{* *}$ & \\
\hline Tanner $2-3$ & $90.10 \pm 9.74$ & $88.76 \pm 9.84$ & $91.13 \pm 9.55$ & \\
\hline Tanner 4-5 & $97.73 \pm 10.32$ & $96.50 \pm 9.96$ & $100.03 \pm 10.82$ & \\
\hline
\end{tabular}
to sex and pubertal stages. 
Table 1. Cont.

\begin{tabular}{|c|c|c|c|c|}
\hline Parameters & All & Females $(n=306)$ & Males $(n=279)$ & $p^{*}$ \\
\hline $\begin{array}{l}\text { Waist } \\
\text { circumference/height ratio }\end{array}$ & $0.58 \pm 0.11$ & $0.58 \pm 0.10$ & $0.57 \pm 0.12$ & \multirow{4}{*}{0.38} \\
\hline Tanner 1 & $0.59 \pm 0.10$ & $0.60 \pm 0.09 * *$ & $0.58 \pm 0.10$ & \\
\hline Tanner 2-3 & $0.57 \pm 0.13$ & $0.56 \pm 0.15$ & $0.58 \pm 0.12$ & \\
\hline Tanner 4-5 & $0.60 \pm 0.06$ & $0.60 \pm 0.06$ & $0.59 \pm 0.06$ & \\
\hline Fat mass (\%) & $37.28 \pm 5.85$ & $37.42 \pm 5.69$ & $36.78 \pm 6.0$ & \multirow{4}{*}{0.06} \\
\hline Tanner 1 & $35.28 \pm 4.88^{* *}$ & $35.86 \pm 4.59 * *$ & $34.35 \pm 5.22 * *$ & \\
\hline Tanner 2-3 & $37.69 \pm 5.77$ & $37.87 \pm 5.78$ & $37.57 \pm 5.78$ & \\
\hline Tanner $4-5$ & $39.15 \pm 7.10$ & $41.06 \pm 5.86$ & $35.52 \pm 7.96$ & \\
\hline Free Fat mass (\%) & $60.98 \pm 9.8$ & $60.25 \pm 9.5$ & $61.77 \pm 10.2$ & \multirow{4}{*}{0.09} \\
\hline Tanner 1 & $61.85 \pm 12.26$ & $62.48 \pm 9.46^{* *}$ & $60.90 \pm 15.67$ & \\
\hline Tanner 2-3 & $61.12 \pm 8.79$ & $59.65 \pm 9.78$ & $62.24 \pm 7.65$ & \\
\hline Tanner 4-5 & $58.81 \pm 9.76$ & $5818 \pm 8.12$ & $60.04 \pm 12.53$ & \\
\hline Visceral Adiposity Index & $3.31 \pm 2.93$ & $3.87 \pm 3.00$ & $2.71 \pm 2.71$ & \multirow{4}{*}{$<0.001$} \\
\hline Tanner 1 & $2.82 \pm 2.16$ & $3.52 \pm 2.42$ & $1.73 \pm 0.95^{* *}$ & \\
\hline Tanner 2-3 & $3.46 \pm 3.26$ & $4.18 \pm 3.38$ & $2.84 \pm 3.03$ & \\
\hline Tanner $4-5$ & $3.60 \pm 2.25$ & $3.34 \pm 2.22$ & $4.04 \pm 2.28$ & \\
\hline Body shape index & $0.078 \pm 0.006$ & $0.077 \pm 0.006$ & $0.079 \pm 0.006$ & \multirow{4}{*}{0.005} \\
\hline Tanner 1 & $0.079 \pm 0.007^{* *}$ & $0.080 \pm 0.005^{* *}$ & $0.078 \pm 0.008$ & \\
\hline Tanner 2-3 & $0.078 \pm 0.005$ & $0.076 \pm 0.005$ & $0.079 \pm 0.005$ & \\
\hline Tanner $4-5$ & $0.075 \pm 0.006$ & $0.075 \pm 0.006$ & $0.077 \pm 0.005$ & \\
\hline Triponderal mass index & $19.32 \pm 2.60$ & $19.61 \pm 2.93$ & $19.01 \pm 2.13$ & \multirow{4}{*}{0.004} \\
\hline Tanner 1 & $19.45 \pm 2.47$ & $19.42 \pm 2.47$ & $19.50 \pm 2.50$ & \\
\hline Tanner 2-3 & $19.20 \pm 2.66$ & $19.56 \pm 3.18$ & $18.90 \pm 2.07$ & \\
\hline Tanner 4-5 & $19.67 \pm 2.47$ & $20.18 \pm 2.75$ & $18.68 \pm 1.39$ & \\
\hline Conicity index & $1.25 \pm 0.09$ & $1.24 \pm 0.09$ & $1.26 \pm 0.09$ & \multirow{4}{*}{0.003} \\
\hline Tanner 1 & $1.25 \pm 0.10$ & $1.25 \pm 0.08$ & $1.23 \pm 0.12^{* *}$ & \\
\hline Tanner 2-3 & $1.25 \pm 0.08$ & $1.23 \pm 0.08$ & $1.27 \pm 0.08$ & \\
\hline Tanner 4-5 & $1.23 \pm 0.10$ & $1.22 \pm 0.10$ & $1.25 \pm 0.09$ & \\
\hline $\begin{array}{l}\text { Homeostasis model } \\
\text { assessment for insulin } \\
\text { resistance }\end{array}$ & $3.42 \pm 2.42$ & $3.58 \pm 2.52$ & $3.24 \pm 2.29$ & \multirow{4}{*}{0.08} \\
\hline Tanner 1 & $2.36 \pm 1.56^{* *}$ & $2.62 \pm 1.73^{* *}$ & $1.96 \pm 1.14 * *$ & \\
\hline Tanner 2-3 & $3.72 \pm 2.38$ & $4.02 \pm 2.63$ & $3.45 \pm 2.11$ & \\
\hline Tanner 4-5 & $4.12 \pm 3.40$ & $3.84 \pm 2.96$ & $4.64 \pm 4.12$ & \\
\hline $\begin{array}{l}\text { Triglyceride and Glucose } \\
\text { index }\end{array}$ & $8.10 \pm 0.46$ & $8.10 \pm 0.46$ & $8.09 \pm 0.53$ & \multirow{4}{*}{0.68} \\
\hline Tanner 1 & $7.97 \pm 0.48^{* *}$ & $8.05 \pm 0.47^{* *}$ & $7.85 \pm 0.49^{* *}$ & \\
\hline Tanner 2-3 & $8.15 \pm 0.49$ & $8.17 \pm 0.45$ & $8.12 \pm 0.53$ & \\
\hline Tanner 4-5 & $8.09 \pm 0.52$ & $7.94 \pm 0.45$ & $8.35 \pm 0.54$ & \\
\hline $\begin{array}{l}\text { HOMA of percentage } \\
\beta \text {-cell function }\end{array}$ & $193.09 \pm 76.67$ & $205.71 \pm 78.49$ & $179.11 \pm 72.21$ & \multirow{4}{*}{$<0.001$} \\
\hline Tanner 1 & $156.10 \pm 63.77^{* *}$ & $168.6 \pm 69.57^{* *}$ & $136.90 \pm 48.22 * *$ & \\
\hline Tanner 2-3 & $203.53 \pm 76.48$ & $222.84 \pm 79.40$ & $186.13 \pm 69.48$ & \\
\hline Tanner $4-5$ & $216.36 \pm 77.74$ & $211.45 \pm 65.91$ & $225.3 \pm 96.73$ & \\
\hline $\begin{array}{l}\text { Quantitative insulin } \\
\text { sensitivity check index }\end{array}$ & $3.05 \pm 0.28$ & $3.08 \pm 0.27$ & $3.03 \pm 0.28$ & \multirow{4}{*}{0.05} \\
\hline Tanner 1 & $2.91 \pm 0.27^{* *}$ & $2.95 \pm 0.28^{* *}$ & $2.83 \pm 0.24^{* *}$ & \\
\hline Tanner 2-3 & $3.11 \pm 0.26$ & $3.14 \pm 0.26$ & 3.080 .26 & \\
\hline Tanner 4-5 & $3.13 \pm 0.28$ & $3.14 \pm 0.27$ & $3.17 \pm 0.30$ & \\
\hline $\begin{array}{l}\text { Fasting blood glucose } \\
(\mathrm{mg} / \mathrm{dL})\end{array}$ & $81.74 \pm 8.92$ & $80.68 \pm 9.34$ & $82.89 \pm 8.29$ & \multirow{4}{*}{0.002} \\
\hline Tanner 1 & $80.75 \pm 8.21$ & $80.58 \pm 7.65$ & $81.01 \pm 9.07$ & \\
\hline Tanner 2-3 & $82.20 \pm 8.79$ & $80.70 \pm 9.68$ & $83.47 \pm 7.71$ & \\
\hline Tanner $4-5$ & $81.21 \pm 10.88$ & $80.51 \pm 11.09$ & $82.59 \pm 10.59$ & \\
\hline Insulin $(\mathrm{mU} / \mathrm{mL})$ & $16.75 \pm 10.46$ & $17.71 \pm 10.93$ & $15.67 \pm 9.81$ & \multirow{4}{*}{0.01} \\
\hline Tanner 1 & $11.71 \pm 7.44^{* *}$ & $13.09 \pm 8.35^{* *}$ & $9.56 \pm 5.09 * *$ & \\
\hline Tanner 2-3 & $18.17 \pm 10.28$ & $19.79 \pm 11.31$ & $16.72 \pm 9.04$ & \\
\hline Tanner $4-5$ & $19.90 \pm 13.41$ & $18.80 \pm 11.44$ & $21.91 \pm 16.51$ & \\
\hline
\end{tabular}


Table 1. Cont.

\begin{tabular}{|c|c|c|c|c|}
\hline Parameters & All & Females $(n=306)$ & Males $(n=279)$ & $p^{*}$ \\
\hline Total cholesterol (mg/dL) & $155.31 \pm 27.61$ & $152.94 \pm 25.92$ & $157.89 \pm 29.18$ & \multirow{4}{*}{0.03} \\
\hline Tanner 1 & $154.65 \pm 26.06$ & $154.52 \pm 27.61$ & $154.85 \pm 23.87^{* *}$ & \\
\hline Tanner 2-3 & $156.21 \pm 27.77$ & $153.65 \pm 24.01$ & $158.47 \pm 30.57$ & \\
\hline Tanner $4-5$ & $151.50 \pm 30.04$ & $146.80 \pm 29.66$ & $160.45 \pm 29.35$ & \\
\hline HDL-cholesterol (mg/dL) & $47.10 \pm 11.10$ & $47.25 \pm 11.45$ & $46.91 \pm 10.71$ & \multirow{4}{*}{0.71} \\
\hline Tanner 1 & $48.89 \pm 12.52 * *$ & $48.52 \pm 12.79$ & $49.46 \pm 1217^{* *}$ & \\
\hline Tanner $2-3$ & $47.04 \pm 10.58$ & $47.28 \pm 11.30$ & $46.83 \pm 9.92$ & \\
\hline Tanner $4-5$ & $43.40 \pm 9.80$ & $44.57 \pm 8.61$ & $41.18 \pm 11.63$ & \\
\hline Tryglicerides (mg/dL) & $92.29 \pm 58.56$ & $92.21 \pm 49.38$ & $92.37 \pm 67.22$ & \multirow{4}{*}{0.97} \\
\hline Tanner 1 & $80.64 \pm 40.82 * *$ & $87.34 \pm 44.76^{* *}$ & $70.33 \pm 31.57 * *$ & \\
\hline Tanner 2-3 & $96.83 \pm 65.06$ & $97.90 \pm 53.03$ & $95.89 \pm 74.17$ & \\
\hline Tanner 4-5 & $91.38 \pm 48.07$ & $78.02 \pm 38.32$ & $116.95 \pm 54.41$ & \\
\hline $\begin{array}{l}\text { Systolic Blood Pressure } \\
(\mathrm{mmHg})\end{array}$ & $111.96 \pm 14.20$ & $111.86 \pm 12.49$ & $112.07 \pm 15.92$ & \multirow{4}{*}{0.43} \\
\hline Tanner 1 & $105.41 \pm 12.20 * *$ & $105.38 \pm 12.35^{* *}$ & $105.47 \pm 11.81 * *$ & \\
\hline Tanner 2-3 & $113.39 \pm 14.28$ & $114.29 \pm 11.29$ & $112.70 \pm 16.39$ & \\
\hline Tanner 4-5 & $117.95 \pm 13.20$ & $115.86 \pm 12.36$ & $122.04 \pm 14.11$ & \\
\hline $\begin{array}{l}\text { Diastolic Blood Pressure } \\
(\mathrm{mmHg})\end{array}$ & $63.62 \pm 9.49$ & $63.68 \pm 8.69$ & $63.50 \pm 10.33$ & \multirow{4}{*}{0.82} \\
\hline Tanner 1 & $60.13 \pm 7.58^{* *}$ & $60.18 \pm 8.21 * *$ & $60.05 \pm 6.47^{* *}$ & \\
\hline Tanner 2-3 & $63.97 \pm 9.66$ & $64.25 \pm 8.22$ & $63.67 \pm 10.74$ & \\
\hline Tanner 4-5 & $69.06 \pm 9.41$ & $68.55 \pm 8.65$ & $70.04 \pm 10.88$ & \\
\hline
\end{tabular}

\subsection{Adiposity Indexes}

Adiposity indexes according to sex and pubertal stages are reported in Table 1 . The BMI-z score did not differ in males and females; in both sexes it was higher in prepuberty than in middle and late puberty $(p<0.001)$.

Compared to females, males had higher WC $(p<0.01)$, ABSI $(p<0.01)$, ConI $(p<0.01)$ and lower VAI and TMI $(p<0.01)$ values, Table 1.

The difference in WC was influenced by puberty in both sexes, with higher values in puberty compared to the prepubertal period $(p<0.001)$. ABSI in prepubertal females was higher compared to girls in middle and late puberty $(p=0.001)$. In males, no differences related to pubertal stage were noted $(p=0.27)$. In both males and females, TMI and ConI did not differ between puberty groups $(p>0.05)$, Table 1 .

VAI in pubertal males was higher compared to boys in middle and late puberty $(p=0.001)$; no significant differences related to pubertal stage were noted in females $(p=0.04)$, Table 1 .

Fat mass and free fat mass did not differ in females and males $(p>0.05)$, Table 1. Fat mass was influenced by puberty in both sexes, with higher values in pubertal stages compared to prepubertal stages $(p<0.01)$. Free fat mass in prepubertal females was higher compared to girls in middle and late puberty $(p=0.001)$, while no differences were related to pubertal stage in boys $(p=0.54)$, Table 1 .

\subsection{Indirect IR Markers}

Concerning IR indexes, lower HOMA- $\beta(p<0.001)$ levels were detected in males compared to females. No other differences in IR indexes were noted between sex groups $(p>0.05)$, Table 1 . The differences in all IR parameters were influenced by puberty in both sexes, with higher values in pubertal patients compared to patients in the prepubertal period $(p<0.001)$.

\subsection{Correlation between IR Measurements and Adiposity Indexes}

As reported in Table 2, in both sexes, WC and VAI significantly correlated with all indirect IR measurements $(p<0.001)$. 
Table 2. Correlation between insulin resistance measurements and adiposity indexes.

\begin{tabular}{|c|c|c|c|c|c|c|c|c|c|c|c|c|}
\hline \multirow{3}{*}{ Adiposity Indexes } & \multicolumn{12}{|c|}{ Indirect Insulin Resistance Measurements } \\
\hline & \multicolumn{3}{|c|}{ HOMA-IR } & \multicolumn{3}{|c|}{ TyG-Index } & \multicolumn{3}{|c|}{ HOMA- $\beta$} & \multicolumn{3}{|c|}{ QUICK Index } \\
\hline & All & Females & Males & All & Females & Males & All & Females & Males & All & Females & Males \\
\hline Body mass index & $\begin{array}{c}\mathrm{r}=0.41 \\
p<0.001\end{array}$ & $\begin{array}{c}\mathrm{r}=0.42 \\
p<0.001\end{array}$ & $\begin{array}{c}r=0.39 \\
p<0.001\end{array}$ & $\begin{aligned} \mathrm{r} & =0.14 \\
p & <0.001\end{aligned}$ & $\begin{array}{l}\mathrm{r}=0.11 \\
p=0.05\end{array}$ & $\begin{aligned} \mathrm{r} & =0.18 \\
p & =0.001\end{aligned}$ & $\begin{array}{l}r=0.45 \\
p<0.001\end{array}$ & $\begin{array}{c}r=0.49 \\
p<0.001\end{array}$ & $\begin{array}{l}\mathrm{r}=0.41 \\
p<0.001\end{array}$ & $\begin{array}{l}\mathrm{r}=0.41 \\
p<0.001\end{array}$ & $\begin{array}{c}r=0.43 \\
p<0.001\end{array}$ & $\begin{aligned} \mathrm{r} & =0.39 \\
p & <0.001\end{aligned}$ \\
\hline Tanner 1 & $\begin{array}{l}r=0.40 \\
p<0.001\end{array}$ & $\begin{array}{c}r=0.49 \\
p<0.001\end{array}$ & $\begin{array}{l}\mathrm{r}=0.26 \\
p=0.04\end{array}$ & $\begin{array}{l}\mathrm{r}=0.16 \\
p=0.05\end{array}$ & $\begin{array}{l}\mathrm{r}=0.23 \\
p=0.03\end{array}$ & $\begin{array}{l}\mathrm{r}=0.16 \\
p=0.24\end{array}$ & $\begin{array}{l}r=0.36 \\
p<0.001\end{array}$ & $\begin{array}{c}r=0.42 \\
p<0.001\end{array}$ & $\begin{array}{l}\mathrm{r}=0.41 \\
p=0.01\end{array}$ & $\begin{array}{l}r=0.31 \\
p<0.001\end{array}$ & $\begin{array}{c}r=0.42 \\
p<0.001\end{array}$ & $\begin{array}{l}\mathrm{r}=0.25 \\
p=0.05\end{array}$ \\
\hline Tanner 2-3 & $\begin{array}{l}r=0.40 \\
p<0.001\end{array}$ & $\begin{array}{c}r=0.46 \\
p<0.001\end{array}$ & $\begin{aligned} r & =0.30 \\
p & <0.001\end{aligned}$ & $\begin{array}{l}\mathrm{r}=0.08 \\
p=0.08\end{array}$ & $\begin{array}{l}\mathrm{r}=0.13 \\
p=0.07\end{array}$ & $\begin{array}{l}\mathrm{r}=0.04 \\
p=0.56\end{array}$ & $\begin{array}{l}\mathrm{r}=0.45 \\
p<0.001\end{array}$ & $\begin{array}{l}r=0.55 \\
p<0.001\end{array}$ & $\begin{array}{l}r=0.30 \\
p<0.001\end{array}$ & $\begin{array}{l}r=0.36 \\
p<0.001\end{array}$ & $\begin{array}{c}r=0.43 \\
p<0.001\end{array}$ & $\begin{aligned} \mathrm{r} & =0.28 \\
p & <0.001\end{aligned}$ \\
\hline Tanner 4-5 & $\begin{array}{l}\mathrm{r}=0.13 \\
p=0.28\end{array}$ & $\begin{array}{c}\mathrm{r}=-0.05 \\
p=0.72\end{array}$ & $\begin{array}{l}\mathrm{r}=0.57 \\
p<0.01\end{array}$ & $\begin{array}{l}\mathrm{r}=0.10 \\
p=0.43\end{array}$ & $\begin{array}{c}\mathrm{r}=-0.02 \\
p=0.88\end{array}$ & $\begin{array}{l}\mathrm{r}=0.53 \\
p=0.01\end{array}$ & $\begin{aligned} \mathrm{r} & =-0.01 \\
p & =0.93\end{aligned}$ & $\begin{array}{c}\mathrm{r}=-0.24 \\
p=0.11\end{array}$ & $\begin{array}{l}\mathrm{r}=0.43 \\
p=0.04\end{array}$ & $\begin{array}{l}\mathrm{r}=0.13 \\
p=0.27\end{array}$ & $\begin{array}{c}\mathrm{r}=-0.13 \\
p=0.93\end{array}$ & $\begin{array}{l}\mathrm{r}=0.48 \\
p=0.02\end{array}$ \\
\hline Body mass index z score & $\begin{array}{c}r=0.19 \\
p<0.001\end{array}$ & $\begin{array}{c}r=0.24 \\
p<0.001\end{array}$ & $\begin{array}{l}r=0.15 \\
p=0.01\end{array}$ & $\begin{array}{c}r=0.18 \\
p<0.001\end{array}$ & $\begin{aligned} r & =0.19 \\
p & <0.001\end{aligned}$ & $\begin{aligned} \mathrm{r} & =0.18 \\
p & =0.002\end{aligned}$ & $\begin{array}{l}\mathrm{r}=0.08 \\
p=0.03\end{array}$ & $\begin{aligned} r & =0.19 \\
p & <0.001\end{aligned}$ & $\begin{array}{c}\mathrm{r}=-0.02 \\
p=0.68\end{array}$ & $\begin{array}{c}r=0.21 \\
p<0.001\end{array}$ & $\begin{array}{c}r=0.27 \\
p<0.001\end{array}$ & $\begin{array}{l}\mathrm{r}=0.15 \\
p=0.01\end{array}$ \\
\hline Tanner 1 & $\begin{array}{c}r=0.34 \\
p<0.001\end{array}$ & $\begin{array}{l}r=0.43 \\
p<0.001\end{array}$ & $\begin{array}{l}\mathrm{r}=0.21 \\
p=0.11\end{array}$ & $\begin{array}{l}\mathrm{r}=0.24 \\
p<0.01\end{array}$ & $\begin{array}{l}\mathrm{r}=0.20 \\
p=0.06\end{array}$ & $\begin{array}{l}\mathrm{r}=0.33 \\
p=0.01\end{array}$ & $\begin{array}{l}\mathrm{r}=0.23 \\
p<0.01\end{array}$ & $\begin{array}{l}r=0.39 \\
p<0.001\end{array}$ & $\begin{array}{c}\mathrm{r}=-0.05 \\
p=0.71\end{array}$ & $\begin{array}{c}\mathrm{r}=0.35 \\
p<0.001\end{array}$ & $\begin{array}{l}r=0.44 \\
p<0.001\end{array}$ & $\begin{array}{l}\mathrm{r}=0.24 \\
p=0.06\end{array}$ \\
\hline Tanner 2-3 & $\begin{aligned} \mathrm{r} & =0.36 \\
p & <0.001\end{aligned}$ & $\begin{aligned} \mathrm{r} & =0.47 \\
p & <0.001\end{aligned}$ & $\begin{aligned} \mathrm{r} & =0.29 \\
p & <0.001\end{aligned}$ & $\begin{aligned} \mathrm{r} & =0.25 \\
p & <0.001\end{aligned}$ & $\begin{array}{l}\mathrm{r}=0.27 \\
p<0.001\end{array}$ & $\begin{array}{l}\mathrm{r}=0.25 \\
p<0.001\end{array}$ & $\begin{array}{l}\mathrm{r}=0.10 \\
p<0.01\end{array}$ & $\begin{aligned} \mathrm{r} & =0.36 \\
p & <0.001\end{aligned}$ & $\begin{array}{l}\mathrm{r}=0.08 \\
p=0.21\end{array}$ & $\begin{aligned} \mathrm{r} & =0.36 \\
p & <0.001\end{aligned}$ & $\begin{aligned} \mathrm{r} & =0.47 \\
p & <0.001\end{aligned}$ & $\begin{aligned} \mathrm{r} & =0.31 \\
p & <0.001\end{aligned}$ \\
\hline Tanner 4-5 & $\begin{array}{l}\mathrm{r}=0.18 \\
p=0.14\end{array}$ & $\begin{array}{c}\mathrm{r}=-0.08 \\
p=0.61\end{array}$ & $\begin{array}{c}r=0.66 \\
p<0.001\end{array}$ & $\begin{array}{l}\mathrm{r}=0.12 \\
p=0.32\end{array}$ & $\begin{aligned} \mathrm{r} & =0.002 \\
p & =0.98\end{aligned}$ & $\begin{array}{l}\mathrm{r}=0.42 \\
p=0.04\end{array}$ & $\begin{aligned} \mathrm{r} & =0.003 \\
p & =0.97\end{aligned}$ & $\begin{array}{c}\mathrm{r}=-0.29 \\
p=0.06\end{array}$ & $\begin{array}{l}\mathrm{r}=0.47 \\
p=0.02\end{array}$ & $\begin{array}{l}\mathrm{r}=0.16 \\
p=0.19\end{array}$ & $\begin{array}{c}\mathrm{r}=-0.02 \\
p=0.89\end{array}$ & $\begin{array}{l}\mathrm{r}=0.58 \\
p<0.01\end{array}$ \\
\hline Waist circumference & $\begin{aligned} r & =0.41 \\
p & <0.001\end{aligned}$ & $\begin{array}{c}r=0.43 \\
p<0.001\end{array}$ & $\begin{array}{c}r=0.42 \\
p<0.001\end{array}$ & $\begin{aligned} \mathrm{r} & =0.20 \\
p & <0.001\end{aligned}$ & $\begin{aligned} \mathrm{r} & =0.15 \\
p & =0.008\end{aligned}$ & $\begin{array}{l}r=0.25 \\
p<0.001\end{array}$ & $\begin{array}{l}r=0.46 \\
p<0.001\end{array}$ & $\begin{array}{l}r=0.53 \\
p<0.001\end{array}$ & $\begin{array}{l}r=0.45 \\
p<0.001\end{array}$ & $\begin{array}{l}r=0.43 \\
p<0.001\end{array}$ & $\begin{array}{c}r=0.44 \\
p<0.001\end{array}$ & $\begin{aligned} \mathrm{r} & =0.44 \\
p & <0.001\end{aligned}$ \\
\hline Tanner 1 & $\begin{array}{l}r=0.35 \\
p<0.001\end{array}$ & $\begin{aligned} \mathrm{r} & =0.50 \\
p & <0.001\end{aligned}$ & $\begin{array}{l}r=0.04 \\
p=0.75\end{array}$ & $\begin{array}{l}\mathrm{r}=0.09 \\
p=0.29\end{array}$ & $\begin{array}{l}\mathrm{r}=0.22 \\
p=0.03\end{array}$ & $\begin{array}{c}\mathrm{r}=-0.07 \\
p=0.60\end{array}$ & $\begin{array}{l}\mathrm{r}=0.31 \\
p<0.001\end{array}$ & $\begin{aligned} \mathrm{r} & =0.41 \\
p & <0.001\end{aligned}$ & $\begin{array}{l}\mathrm{r}=0.13 \\
p=0.33\end{array}$ & $\begin{aligned} \mathrm{r} & =0.30 \\
p & <0.001\end{aligned}$ & $\begin{aligned} r & =0.41 \\
p & <0.001\end{aligned}$ & $\begin{array}{l}\mathrm{r}=0.11 \\
p=0.39\end{array}$ \\
\hline Tanner 2-3 & $\begin{array}{c}r=0.39 \\
p<0.001\end{array}$ & $\begin{aligned} r & =0.48 \\
p & <0.001\end{aligned}$ & $\begin{array}{l}r=0.34 \\
p<0.001\end{array}$ & $\begin{array}{l}r=0.14 \\
p<0.01\end{array}$ & $\begin{array}{l}\mathrm{r}=0.16 \\
p=0.03\end{array}$ & $\begin{array}{l}\mathrm{r}=0.14 \\
p=0.04\end{array}$ & $\begin{array}{l}\mathrm{r}=0.44 \\
p<0.001\end{array}$ & $\begin{aligned} r & =0.60 \\
p & <0.001\end{aligned}$ & $\begin{array}{l}r=0.38 \\
p<0.001\end{array}$ & $\begin{aligned} r & =0.39 \\
p & <0.001\end{aligned}$ & $\begin{array}{l}\mathrm{r}=0.47 \\
p<0.001\end{array}$ & $\begin{aligned} \mathrm{r} & =0.34 \\
p & <0.001\end{aligned}$ \\
\hline Tanner $4-5$ & $\begin{array}{l}\mathrm{r}=0.16 \\
p=0.20\end{array}$ & $\begin{array}{c}\mathrm{r}=-0.08 \\
p=0.59\end{array}$ & $\begin{array}{c}r=0.41 \\
p<0.001\end{array}$ & $\begin{array}{c}r=0.42 \\
p<0.001\end{array}$ & $\begin{array}{l}\mathrm{r}=0.25 \\
p=0.12\end{array}$ & $\begin{aligned} r & =0.54 \\
p & <0.001\end{aligned}$ & $\begin{array}{l}\mathrm{r}=0.22 \\
p=0.08\end{array}$ & $\begin{aligned} \mathrm{r} & =-0.06 \\
p & =0.71\end{aligned}$ & $\begin{array}{l}\mathrm{r}=0.36 \\
p=0.09\end{array}$ & $\begin{array}{l}\mathrm{r}=0.13 \\
p=0.31\end{array}$ & $\begin{array}{c}\mathrm{r}=-0.01 \\
p=0.94\end{array}$ & $\begin{array}{l}\mathrm{r}=0.29 \\
p=0.17\end{array}$ \\
\hline $\begin{array}{l}\text { Waist circumference/height } \\
\text { ratio }\end{array}$ & $\begin{array}{l}r=0.08 \\
p=0.05\end{array}$ & $\begin{array}{l}r=0.07 \\
p=0.18\end{array}$ & $\begin{array}{l}\mathrm{r}=0.10 \\
p=0.09\end{array}$ & $\begin{array}{l}\mathrm{r}=0.07 \\
p=0.08\end{array}$ & $\begin{array}{l}\mathrm{r}=0.07 \\
p=0.17\end{array}$ & $\begin{array}{l}r=0.06 \\
p=0.26\end{array}$ & $\begin{array}{l}\mathrm{r}=0.07 \\
p=0.07\end{array}$ & $\begin{array}{l}\mathrm{r}=0.11 \\
p=0.04\end{array}$ & $\begin{array}{l}\mathrm{r}=0.14 \\
p=0.01\end{array}$ & $\begin{array}{l}\mathrm{r}=0.07 \\
p=0.07\end{array}$ & $\begin{array}{l}\mathrm{r}=0.06 \\
p=0.26\end{array}$ & $\begin{array}{l}\mathrm{r}=0.10 \\
p=0.08\end{array}$ \\
\hline Tanner 1 & $\begin{array}{l}r=0.14 \\
p=0.09\end{array}$ & $\begin{array}{l}r=0.22 \\
p=0.03\end{array}$ & $\begin{array}{c}\mathrm{r}=-0.05 \\
p=0.66\end{array}$ & $\begin{aligned} \mathrm{r} & =0.009 \\
p & =0.90\end{aligned}$ & $\begin{array}{l}\mathrm{r}=0.04 \\
p=0.67\end{array}$ & $\begin{array}{c}\mathrm{r}=-0.06 \\
p=0.63\end{array}$ & $\begin{array}{l}\mathrm{r}=0.16 \\
p=0.04\end{array}$ & $\begin{array}{l}\mathrm{r}=0.23 \\
p=0.03\end{array}$ & $\begin{array}{l}\mathrm{r}=0.01 \\
p=0.90\end{array}$ & $\begin{array}{l}\mathrm{r}=0.09 \\
p=0.28\end{array}$ & $\begin{array}{l}\mathrm{r}=0.13 \\
p=0.23\end{array}$ & $\begin{array}{c}\mathrm{r}=-0.009 \\
p=0.944\end{array}$ \\
\hline Tanner 2-3 & $\begin{array}{l}\mathrm{r}=0.08 \\
p=0.08\end{array}$ & $\begin{array}{l}\mathrm{r}=0.10 \\
p=0.15\end{array}$ & $\begin{array}{l}\mathrm{r}=0.09 \\
p=0.17\end{array}$ & $\begin{array}{l}\mathrm{r}=0.08 \\
p=0.11\end{array}$ & $\begin{array}{l}\mathrm{r}=0.11 \\
p=0.14\end{array}$ & $\begin{array}{l}\mathrm{r}=0.06 \\
p=0.34\end{array}$ & $\begin{array}{l}\mathrm{r}=0.12 \\
p=0.01\end{array}$ & $\begin{array}{l}\mathrm{r}=0.16 \\
p=0.02\end{array}$ & $\begin{array}{l}\mathrm{r}=0.15 \\
p=0.02\end{array}$ & $\begin{array}{l}\mathrm{r}=0.09 \\
p=0.07\end{array}$ & $\begin{array}{l}\mathrm{r}=0.10 \\
p=0.15\end{array}$ & $\begin{array}{l}\mathrm{r}=0.11 \\
p=0.11\end{array}$ \\
\hline Tanner 4-5 & $\begin{aligned} \mathrm{r} & =0.312 \\
p & =0.32\end{aligned}$ & $\begin{array}{c}\mathrm{r}=-0.05 \\
p=0.74\end{array}$ & $\begin{array}{l}\mathrm{r}=0.40 \\
p=0.06\end{array}$ & $\begin{aligned} \mathrm{r} & =0.37 \\
p & =0.003\end{aligned}$ & $\begin{array}{c}\mathrm{r}=-0.31 \\
p=0.05\end{array}$ & $\begin{array}{l}\mathrm{r}=0.58 \\
p<0.01\end{array}$ & $\begin{array}{l}\mathrm{r}=0.11 \\
p=0.38\end{array}$ & $\begin{array}{c}\mathrm{r}=-0.04 \\
p=0.78\end{array}$ & $\begin{array}{l}\mathrm{r}=0.34 \\
p=0.12\end{array}$ & $\begin{array}{l}\mathrm{r}=0.17 \\
p=0.17\end{array}$ & $\begin{array}{c}\mathrm{r}=-0.08 \\
p=0.61\end{array}$ & $\begin{array}{l}\mathrm{r}=0.34 \\
p=0.11\end{array}$ \\
\hline Fat mass & $\begin{array}{c}r=0.18 \\
p<0.001\end{array}$ & $\begin{array}{c}r=0.27 \\
p<0.001\end{array}$ & $\begin{array}{l}\mathrm{r}=0.05 \\
p=0.38\end{array}$ & $\begin{aligned} \mathrm{r} & =0.12 \\
p & =0.006\end{aligned}$ & $\begin{array}{l}\mathrm{r}=0.13 \\
p=0.02\end{array}$ & $\begin{array}{l}r=0.10 \\
p=0.10\end{array}$ & $\begin{aligned} \mathrm{r} & =0.18 \\
p & <0.001\end{aligned}$ & $\begin{array}{l}r=0.25 \\
p<0.001\end{array}$ & $\begin{array}{l}\mathrm{r}=0.08 \\
p=0.18\end{array}$ & $\begin{aligned} r & =0.19 \\
p & <0.001\end{aligned}$ & $\begin{aligned} r & =0.30 \\
p & <0.001\end{aligned}$ & $\begin{array}{l}\mathrm{r}=0.04 \\
p=0.48\end{array}$ \\
\hline Tanner 1 & $\begin{array}{l}r=0.26 \\
p<0.01\end{array}$ & $\begin{array}{l}r=0.31 \\
p<0.01\end{array}$ & $\begin{array}{l}\mathrm{r}=0.12 \\
p=0.40\end{array}$ & $\begin{array}{l}\mathrm{r}=0.23 \\
p<0.01\end{array}$ & $\begin{array}{l}\mathrm{r}=0.18 \\
p=0.10\end{array}$ & $\begin{array}{c}\mathrm{r}=-0.25 \\
p=0.07\end{array}$ & $\begin{array}{l}\mathrm{r}=0.20 \\
p=0.02\end{array}$ & $\begin{array}{l}\mathrm{r}=0.23 \\
p=0.03\end{array}$ & $\begin{array}{l}\mathrm{r}=0.06 \\
p=0.67\end{array}$ & $\begin{array}{l}\mathrm{r}=0.23 \\
p<0.01\end{array}$ & $\begin{array}{l}\mathrm{r}=0.27 \\
p=0.01\end{array}$ & $\begin{array}{l}\mathrm{r}=0.11 \\
p=0.42\end{array}$ \\
\hline
\end{tabular}


Table 2. Cont.

\begin{tabular}{|c|c|c|c|c|c|c|c|c|c|c|c|c|}
\hline \multirow{3}{*}{ Adiposity Indexes } & \multicolumn{12}{|c|}{ Indirect Insulin Resistance Measurements } \\
\hline & \multicolumn{3}{|c|}{ HOMA-IR } & \multicolumn{3}{|c|}{ TyG-Index } & \multicolumn{3}{|c|}{ HOMA- $\beta$} & \multicolumn{3}{|c|}{ QUICK Index } \\
\hline & All & Females & Males & All & Females & Males & All & Females & Males & All & Females & Males \\
\hline Tanner 2-3 & $\begin{aligned} r & =0.19 \\
p & <0.001\end{aligned}$ & $\begin{array}{c}r=0.34 \\
p<0.001\end{array}$ & $\begin{array}{l}\mathrm{r}=0.01 \\
p=0.88\end{array}$ & $\begin{array}{l}\mathrm{r}=0.05 \\
p=0.29\end{array}$ & $\begin{array}{l}\mathrm{r}=0.13 \\
p=0.08\end{array}$ & $\begin{array}{c}\mathrm{r}=-0.003 \\
p=0.96\end{array}$ & $\begin{array}{l}\mathrm{r}=0.16 \\
p<0.01\end{array}$ & $\begin{array}{c}r=0.28 \\
p<0.001\end{array}$ & $\begin{array}{l}\mathrm{r}=0.03 \\
p=0.66\end{array}$ & $\begin{array}{l}r=0.14 \\
p<0.001\end{array}$ & $\begin{array}{l}r=0.37 \\
p<0.001\end{array}$ & $\begin{array}{c}\mathrm{r}=-0.06 \\
p=0.37\end{array}$ \\
\hline Tanner 4-5 & $\begin{array}{c}\mathrm{r}=-0.14 \\
p=0.30\end{array}$ & $\begin{array}{l}\mathrm{r}=-0.20 \\
p=0.721\end{array}$ & $\begin{array}{c}\mathrm{r}=-0.002 \\
p=0.99\end{array}$ & $\begin{array}{l}\mathrm{r}=0.07 \\
p=0.60\end{array}$ & $\begin{array}{c}\mathrm{r}=-0.13 \\
p=0.43\end{array}$ & $\begin{array}{l}\mathrm{r}=0.34 \\
p=0.13\end{array}$ & $\begin{array}{c}\mathrm{r}=-0.07 \\
p=0.60\end{array}$ & $\begin{array}{c}\mathrm{r}=-0.18 \\
p=0.29\end{array}$ & $\begin{array}{l}\mathrm{r}=0.06 \\
p=0.80\end{array}$ & $\begin{array}{c}\mathrm{r}=-0.04 \\
p=0.73\end{array}$ & $\begin{array}{l}\mathrm{r}=-0.11 \\
p=0.452\end{array}$ & $\begin{array}{l}\mathrm{r}=0.10 \\
p=0.65\end{array}$ \\
\hline Free Fat mass & $\begin{array}{l}\mathrm{r}=-0.18 \\
p=0.004\end{array}$ & $\begin{array}{l}\mathrm{r}=-0.22 \\
p<0.001\end{array}$ & $\begin{array}{c}\mathrm{r}=-0.004 \\
p=0.95\end{array}$ & $\begin{array}{c}\mathrm{r}=-0.04 \\
p=0.30\end{array}$ & $\begin{array}{c}\mathrm{r}=-0.002 \\
p=0.97\end{array}$ & $\begin{array}{c}\mathrm{r}=-0.08 \\
p=0.22\end{array}$ & $\begin{array}{c}\mathrm{r}=-0.03 \\
p=0.43\end{array}$ & $\begin{array}{c}\mathrm{r}=-0.12 \\
p=0.06\end{array}$ & $\begin{array}{l}\mathrm{r}=0.08 \\
p=0.22\end{array}$ & $\begin{array}{c}\mathrm{r}=-0.10 \\
p=0.03\end{array}$ & $\begin{array}{l}\mathrm{r}=-0.19 \\
p=0.002\end{array}$ & $\begin{array}{l}\mathrm{r}=0.007 \\
p=0.91\end{array}$ \\
\hline Tanner 1 & $\begin{array}{l}\mathrm{r}=0.01 \\
p=0.86\end{array}$ & $\begin{array}{c}\mathrm{r}=-0.01 \\
p=0.88\end{array}$ & $\begin{array}{l}\mathrm{r}=0.03 \\
p=0.79\end{array}$ & $\begin{array}{c}\mathrm{r}=-0.04 \\
p=0.41\end{array}$ & $\begin{array}{l}\mathrm{r}=0.13 \\
p=0.27\end{array}$ & $\begin{array}{c}\mathrm{r}=-0.22 \\
p=0.12\end{array}$ & $\begin{array}{l}\mathrm{r}=0.05 \\
p=0.56\end{array}$ & $\begin{array}{c}\mathrm{r}=0.0001 \\
p=0.99\end{array}$ & $\begin{array}{l}\mathrm{r}=0.11 \\
p=0.43\end{array}$ & $\begin{array}{l}\mathrm{r}=0.08 \\
p=0.37\end{array}$ & $\begin{array}{l}\mathrm{r}=0.10 \\
p=0.41\end{array}$ & $\begin{array}{l}\mathrm{r}=0.05 \\
p=0.70\end{array}$ \\
\hline Tanner 2-3 & $\begin{array}{l}\mathrm{r}=-0.23 \\
p<0.001\end{array}$ & $\begin{array}{l}\mathrm{r}=-0.31 \\
p<0.001\end{array}$ & $\begin{array}{c}\mathrm{r}=-0.06 \\
p=0.44\end{array}$ & $\begin{array}{c}\mathrm{r}=-0.04 \\
p=0.41\end{array}$ & $\begin{array}{c}\mathrm{r}=-0.05 \\
p=0.51\end{array}$ & $\begin{array}{c}\mathrm{r}=-0.01 \\
p=0.82\end{array}$ & $\begin{array}{c}\mathrm{r}=-0.08 \\
p=0.13\end{array}$ & $\begin{array}{c}\mathrm{r}=-0.15 \\
p=0.06\end{array}$ & $\begin{array}{l}\mathrm{r}=0.07 \\
p=0.38\end{array}$ & $\begin{array}{c}\mathrm{r}=-0.19 \\
p<0.01\end{array}$ & $\begin{array}{l}\mathrm{r}=-0.30 \\
p<0.001\end{array}$ & $\begin{array}{c}\mathrm{r}=-0.03 \\
p=0.66\end{array}$ \\
\hline Tanner 4-5 & $\begin{array}{l}\mathrm{r}=0.04 \\
p=0.72\end{array}$ & $\begin{array}{c}\mathrm{r}=-0.02 \\
p=0.87\end{array}$ & $\begin{array}{c}\mathrm{r}=-0.09 \\
p=0.70\end{array}$ & $\begin{aligned} \mathrm{r}=-0.03 \\
p=0.82\end{aligned}$ & $\begin{array}{c}\mathrm{r}=-0.04 \\
p=0.78\end{array}$ & $\begin{array}{c}\mathrm{r}=-0.07 \\
p=0.74\end{array}$ & $\begin{array}{l}\mathrm{r}=0.13 \\
p=0.32\end{array}$ & $\begin{array}{l}\mathrm{r}=0.12 \\
p=0.46\end{array}$ & $\begin{array}{l}\mathrm{r}=0.14 \\
p=0.56\end{array}$ & $\begin{array}{c}\mathrm{r}=-0.05 \\
p=0.64\end{array}$ & $\begin{array}{c}\mathrm{r}=-0.16 \\
p=0.35\end{array}$ & $\begin{array}{c}\mathrm{r}=-0.02 \\
p=0.92\end{array}$ \\
\hline VAI & $\begin{array}{c}\mathrm{r}=0.26 \\
p<0.001\end{array}$ & $\begin{array}{l}r=0.21 \\
p<0.001\end{array}$ & $\begin{array}{l}\mathrm{r}=0.32 \\
p<0.001\end{array}$ & $\begin{array}{l}\mathrm{r}=0.77 \\
p<0.001\end{array}$ & $\begin{array}{l}r=0.79 \\
p<0.001\end{array}$ & $\begin{array}{l}r=0.80 \\
p<0.001\end{array}$ & $\begin{array}{l}r=0.32 \\
p<0.001\end{array}$ & $\begin{array}{l}r=0.27 \\
p<0.001\end{array}$ & $\begin{array}{l}r=0.32 \\
p<0.001\end{array}$ & $\begin{array}{l}r=0.29 \\
p<0.001\end{array}$ & $\begin{array}{l}r=0.24 \\
p<0.001\end{array}$ & $\begin{aligned} \mathrm{r} & =0.33 \\
p & <0.001\end{aligned}$ \\
\hline Tanner 1 & $\begin{aligned} \mathrm{r} & =0.30 \\
p & <0.001\end{aligned}$ & $\begin{array}{c}\mathrm{r}=0.33 \\
p=0.001\end{array}$ & $\begin{array}{l}\mathrm{r}=0.38 \\
p<0.01\end{array}$ & $\begin{array}{l}r=0.80 \\
p<0.001\end{array}$ & $\begin{array}{l}r=0.87 \\
p<0.001\end{array}$ & $\begin{array}{l}r=0.88 \\
p<0.001\end{array}$ & $\begin{array}{l}r=0.39 \\
p<0.001\end{array}$ & $\begin{array}{l}r=0.38 \\
p<0.001\end{array}$ & $\begin{array}{l}\mathrm{r}=0.12 \\
p=0.35\end{array}$ & $\begin{array}{l}r=0.38 \\
p<0.001\end{array}$ & $\begin{aligned} r & =0.33 \\
p & =0.001\end{aligned}$ & $\begin{aligned} \mathrm{r} & =0.41 \\
p & =0.001\end{aligned}$ \\
\hline Tanner 2-3 & $\mathrm{r}=0.27$ & $\mathrm{r}=0.22$ & $\mathrm{r}=0.31$ & $\mathrm{r}=0.78$ & $\mathrm{r}=0.77$ & $\mathrm{r}=0.81$ & $\mathrm{r}=0.31$ & $\mathrm{r}=0.23$ & $\mathrm{r}=0.32$ & $\mathrm{r}=0.28$ & $\mathrm{r}=0.23$ & $\mathrm{r}=0.30$ \\
\hline Tanner 4-5 & $\begin{array}{l}\mathrm{r}=0.01 \\
p=0.93\end{array}$ & $\begin{array}{c}\mathrm{r}=-0.10 \\
p=0.54\end{array}$ & $\begin{array}{l}\mathrm{r}=0.12 \\
p=0.58\end{array}$ & $\begin{array}{l}r=0.84 \\
p<0.001\end{array}$ & $\begin{array}{l}r=0.84 \\
p<0.001\end{array}$ & $\begin{array}{l}r=0.88 \\
p<0.001\end{array}$ & $\begin{array}{l}\mathrm{r}=0.09 \\
p=0.46\end{array}$ & $\begin{array}{l}\mathrm{r}=0.10 \\
p=0.54\end{array}$ & $\begin{array}{l}\mathrm{r}=0.06 \\
p=0.79\end{array}$ & $\begin{array}{l}r=0.07 \\
p<0.001\end{array}$ & $\begin{aligned} \mathrm{r} & =0.001 \\
p & =0.99\end{aligned}$ & $\begin{array}{c}\mathrm{r}=0.15 \\
p=0.050\end{array}$ \\
\hline ABSI & $\begin{array}{c}\mathrm{r}=-0.04 \\
p=0.24\end{array}$ & $\begin{array}{c}\mathrm{r}=-0.05 \\
p=0.32\end{array}$ & $\begin{array}{c}\mathrm{r}=-0.02 \\
p=0.68\end{array}$ & $\begin{array}{l}\mathrm{r}=0.07 \\
p=0.07\end{array}$ & $\begin{array}{l}\mathrm{r}=0.10 \\
p=0.07\end{array}$ & $\begin{array}{l}\mathrm{r}=0.04 \\
p=0.42\end{array}$ & $\begin{array}{c}\mathrm{r}=-0.02 \\
p=0.56\end{array}$ & $\begin{array}{c}\mathrm{r}=-0.004 \\
p=0.93\end{array}$ & $\begin{array}{c}\mathrm{r}=-0.009 \\
p=0.87\end{array}$ & $\begin{array}{c}\mathrm{r}=-0.05 \\
p=0.16\end{array}$ & $\begin{array}{c}\mathrm{r}=-0.07 \\
p=0.21\end{array}$ & $\begin{array}{c}\mathrm{r}=-0.02 \\
p=0.64\end{array}$ \\
\hline Tanner 1 & $\begin{array}{c}\mathrm{r}=-0.03 \\
p=0.65\end{array}$ & $\begin{array}{l}\mathrm{r}=0.02 \\
p=0.79\end{array}$ & $\begin{array}{c}\mathrm{r}=-0.21 \\
p=0.10\end{array}$ & $\begin{aligned} \mathrm{r}=-0.12 \\
p=0.15\end{aligned}$ & $\begin{array}{c}\mathrm{r}=-0.07 \\
p=0.47\end{array}$ & $\begin{array}{c}\mathrm{r}=-0.21 \\
p=0.11\end{array}$ & $\begin{array}{c}\mathrm{r}=-0.04 \\
p=0.56\end{array}$ & $\begin{array}{l}\mathrm{r}=0.01 \\
p=0.90\end{array}$ & $\begin{array}{c}\mathrm{r}=-0.23 \\
p=0.08\end{array}$ & $\begin{array}{c}\mathrm{r}=-0.07 \\
p=0.38\end{array}$ & $\begin{array}{c}\mathrm{r}=-0.06 \\
p=0.58\end{array}$ & $\begin{array}{c}\mathrm{r}=-0.16 \\
p=0.23\end{array}$ \\
\hline Tanner 2-3 & $\begin{array}{c}\mathrm{r}=-0.01 \\
p=0.76\end{array}$ & $\begin{array}{l}\mathrm{r}=0.01 \\
p=0.84\end{array}$ & $\begin{array}{l}\mathrm{r}=0.001 \\
p=0.98\end{array}$ & $\begin{array}{l}\mathrm{r}=0.13 \\
p=0.01\end{array}$ & $\begin{array}{l}\mathrm{r}=0.15 \\
p=0.04\end{array}$ & $\begin{array}{l}\mathrm{r}=0.15 \\
p=0.03\end{array}$ & $\begin{array}{l}\mathrm{r}=0.01 \\
p=0.84\end{array}$ & $\begin{array}{l}\mathrm{r}=0.07 \\
p=0.31\end{array}$ & $\begin{array}{l}\mathrm{r}=0.05 \\
p=0.48\end{array}$ & $\begin{array}{c}\mathrm{r}=-0.001 \\
p=0.99\end{array}$ & $\begin{array}{l}\mathrm{r}=0.03 \\
p=0.65\end{array}$ & $\begin{array}{l}\mathrm{r}=0.01 \\
p=0.84\end{array}$ \\
\hline Tanner 4-5 & $\begin{array}{l}\mathrm{r}=0.04 \\
p=0.72\end{array}$ & $\begin{array}{c}\mathrm{r}=-0.01 \\
p=0.92\end{array}$ & $\begin{aligned} r & =0.10 \\
p & =0.065\end{aligned}$ & $\begin{array}{l}\mathrm{r}=0.38 \\
p<0.01\end{array}$ & $\begin{array}{l}\mathrm{r}=0.34 \\
p=0.03\end{array}$ & $\begin{array}{l}\mathrm{r}=0.35 \\
p=0.10\end{array}$ & $\begin{array}{l}\mathrm{r}=0.21 \\
p=0.10\end{array}$ & $\begin{array}{l}\mathrm{r}=0.25 \\
p=0.12\end{array}$ & $\begin{array}{l}\mathrm{r}=0.13 \\
p=0.53\end{array}$ & $\begin{array}{l}\mathrm{r}=0.06 \\
p=0.62\end{array}$ & $\begin{array}{l}\mathrm{r}=0.04 \\
p=0.77\end{array}$ & $\begin{array}{l}\mathrm{r}=0.05 \\
p=0.82\end{array}$ \\
\hline TMI & $\begin{array}{c}r=0.23 \\
p<0.001\end{array}$ & $\begin{aligned} r & =0.30 \\
p & <0.001\end{aligned}$ & $\begin{array}{l}\mathrm{r}=0.07 \\
p=0.06\end{array}$ & $\begin{array}{l}\mathrm{r}=0.07 \\
p=0.06\end{array}$ & $\begin{array}{l}\mathrm{r}=0.11 \\
p=0.04\end{array}$ & $\begin{array}{l}\mathrm{r}=0.02 \\
p=0.65\end{array}$ & $\begin{array}{l}r=0.25 \\
p<0.001\end{array}$ & $\begin{array}{c}r=0.33 \\
p<0.001\end{array}$ & $\begin{array}{l}\mathrm{r}=0.09 \\
p=0.10\end{array}$ & $\begin{array}{c}r=0.18 \\
p<0.001\end{array}$ & $\begin{array}{c}r=0.26 \\
p<0.001\end{array}$ & $\begin{aligned} \mathrm{r}=-0.03 \\
p=0.55\end{aligned}$ \\
\hline Tanner 1 & $\begin{array}{l}\mathrm{r}=0.18 \\
p=0.02\end{array}$ & $\begin{array}{l}\mathrm{r}=0.29 \\
p<0.01\end{array}$ & $\begin{array}{c}\mathrm{r}=-0.02 \\
p=0.83\end{array}$ & $\begin{array}{l}\mathrm{r}=0.03 \\
p=0.72\end{array}$ & $\begin{array}{l}\mathrm{r}=0.06 \\
p=0.54\end{array}$ & $\begin{array}{c}\mathrm{r}=-0.003 \\
p=0.97\end{array}$ & $\begin{array}{l}\mathrm{r}=0.20 \\
p=0.01\end{array}$ & $\begin{array}{l}\mathrm{r}=0.25 \\
p=0.01\end{array}$ & $\begin{array}{l}\mathrm{r}=0.13 \\
p=0.31\end{array}$ & $\begin{array}{l}\mathrm{r}=0.12 \\
p=0.14\end{array}$ & $\begin{array}{l}\mathrm{r}=0.22 \\
p=0.03\end{array}$ & $\begin{array}{c}\mathrm{r}=-0.04 \\
p=0.74\end{array}$ \\
\hline Tanner 2-3 & $\begin{array}{l}r=0.30 \\
p<0.001\end{array}$ & $\begin{array}{c}r=0.38 \\
p<0.001\end{array}$ & $\begin{array}{l}\mathrm{r}=0.13 \\
p=0.06\end{array}$ & $\begin{array}{l}\mathrm{r}=0.11 \\
p=0.03\end{array}$ & $\begin{array}{l}\mathrm{r}=0.17 \\
p=0.02\end{array}$ & $\begin{array}{l}\mathrm{r}=0.04 \\
p=0.56\end{array}$ & $\begin{array}{l}r=0.35 \\
p<0.001\end{array}$ & $\begin{array}{c}r=0.47 \\
p<0.001\end{array}$ & $\begin{array}{l}\mathrm{r}=0.13 \\
p=0.06\end{array}$ & $\begin{array}{l}r=0.23 \\
p<0.001\end{array}$ & $\begin{array}{c}r=0.33 \\
p<0.001\end{array}$ & $\begin{array}{l}\mathrm{r}=0.09 \\
p=0.20\end{array}$ \\
\hline Tanner 4-5 & $\begin{array}{l}\mathrm{r}=0.09 \\
p=0.48\end{array}$ & $\begin{array}{c}\mathrm{r}=-0.03 \\
p=0.84\end{array}$ & $\begin{array}{l}r=0.58 \\
p<0.01\end{array}$ & $\begin{array}{l}\mathrm{r}=0.01 \\
p=0.87\end{array}$ & $\begin{array}{l}\mathrm{r}=0.003 \\
p=0.98\end{array}$ & $\begin{aligned} \mathrm{r} & =0.58 \\
p & =0.004\end{aligned}$ & $\begin{array}{c}\mathrm{r}=-0.11 \\
p=0.38\end{array}$ & $\begin{array}{l}\mathrm{r}=-0.31 \\
p=0.704\end{array}$ & $\begin{array}{l}\mathrm{r}=0.41 \\
p=0.05\end{array}$ & $\begin{array}{l}\mathrm{r}=0.15 \\
p=0.23\end{array}$ & $\begin{array}{l}\mathrm{r}=0.07 \\
p=0.63\end{array}$ & $\begin{array}{l}r=0.58 \\
p<0.01\end{array}$ \\
\hline
\end{tabular}


Table 2. Cont.

\begin{tabular}{|c|c|c|c|c|c|c|c|c|c|c|c|c|}
\hline \multirow{3}{*}{ Adiposity Indexes } & \multicolumn{12}{|c|}{ Indirect Insulin Resistance Measurements } \\
\hline & \multicolumn{3}{|c|}{ HOMA-IR } & \multicolumn{3}{|c|}{ TyG-Index } & \multicolumn{3}{|c|}{ НОМА- $\beta$} & \multicolumn{3}{|c|}{ QUICK Index } \\
\hline & All & Females & Males & All & Females & Males & All & Females & Males & All & Females & Males \\
\hline ConI & $\begin{array}{l}\mathrm{r}=0.08 \\
p=0.05\end{array}$ & $\begin{array}{l}\mathrm{r}=0.09 \\
p=0.11\end{array}$ & $\begin{array}{l}\mathrm{r}=0.09 \\
p=0.13\end{array}$ & $\begin{aligned} \mathrm{r} & =0.12 \\
p & =0.003\end{aligned}$ & $\begin{array}{l}\mathrm{r}=0.15 \\
p=0.01\end{array}$ & $\begin{array}{l}\mathrm{r}=0.10 \\
p=0.07\end{array}$ & $\begin{aligned} \mathrm{r} & =0.17 \\
p & =0.003\end{aligned}$ & $\begin{array}{l}\mathrm{r}=0.11 \\
p=0.06\end{array}$ & $\begin{array}{l}\mathrm{r}=0.09 \\
p=0.10\end{array}$ & $\begin{array}{l}\mathrm{r}=0.07 \\
p=0.08\end{array}$ & $\begin{array}{l}\mathrm{r}=0.07 \\
p=0.18\end{array}$ & $\begin{array}{c}\mathrm{r}=-0.08 \\
p=0.15\end{array}$ \\
\hline Tanner 1 & $\begin{array}{l}r=0.04 \\
p=0.61\end{array}$ & $\begin{array}{l}\mathrm{r}=0.16 \\
p=0.12\end{array}$ & $\begin{array}{c}\mathrm{r}=-0.20 \\
p=0.13\end{array}$ & $\begin{array}{c}\mathrm{r}=-0.09 \\
p=0.27\end{array}$ & $\begin{array}{c}\mathrm{r}=-0.01 \\
p=0.86\end{array}$ & $\begin{array}{c}\mathrm{r}=-0.21 \\
p=0.11\end{array}$ & $\begin{array}{l}r=0.02 \\
p=0.75\end{array}$ & $\begin{array}{l}\mathrm{r}=0.12 \\
p=0.24\end{array}$ & $\begin{array}{c}\mathrm{r}=-0.19 \\
p=0.15\end{array}$ & $\begin{array}{c}\mathrm{r}=-0.005 \\
p=0.95\end{array}$ & $\begin{array}{l}\mathrm{r}=0.06 \\
p=0.57\end{array}$ & $\begin{array}{c}\mathrm{r}=-0.14 \\
p=0.29\end{array}$ \\
\hline Tanner 2-3 & $\begin{array}{l}\mathrm{r}=0.11 \\
p=0.03\end{array}$ & $\begin{array}{l}\mathrm{r}=0.17 \\
p=0.02\end{array}$ & $\begin{array}{l}\mathrm{r}=0.10 \\
p=0.16\end{array}$ & $\begin{array}{l}\mathrm{r}=0.16 \\
p<0.01\end{array}$ & $\begin{array}{l}\mathrm{r}=0.19 \\
p=0.01\end{array}$ & $\begin{array}{l}\mathrm{r}=0.17 \\
p=0.01\end{array}$ & $\begin{array}{l}\mathrm{r}=0.15 \\
p<0.01\end{array}$ & $\begin{aligned} \mathrm{r} & =0.26 \\
p & <0.001\end{aligned}$ & $\begin{array}{l}\mathrm{r}=0.15 \\
p=0.03\end{array}$ & $\begin{array}{l}\mathrm{r}=0.11 \\
p=0.02\end{array}$ & $\begin{array}{l}\mathrm{r}=0.18 \\
p=0.02\end{array}$ & $\begin{array}{l}\mathrm{r}=0.10 \\
p=0.14\end{array}$ \\
\hline Tanner 4-5 & $\begin{array}{l}\mathrm{r}=0.07 \\
p=0.54\end{array}$ & $\begin{array}{c}\mathrm{r}=-0.03 \\
p=0.83\end{array}$ & $\begin{array}{l}\mathrm{r}=0.20 \\
p=0.34\end{array}$ & $\begin{aligned} \mathrm{r} & =0.41 \\
p & <0.001\end{aligned}$ & $\begin{array}{c}\mathrm{r}=-0.35 \\
p=0.02\end{array}$ & $\begin{array}{l}\mathrm{r}=0.44 \\
p=0.03\end{array}$ & $\begin{array}{l}\mathrm{r}=0.21 \\
p=0.09\end{array}$ & $\begin{array}{l}\mathrm{r}=0.20 \\
p=0.21\end{array}$ & $\begin{array}{l}\mathrm{r}=0.21 \\
p=0.33\end{array}$ & $\begin{array}{l}\mathrm{r}=0.09 \\
p=0.47\end{array}$ & $\begin{array}{c}\mathrm{r}=-0.04 \\
p=0.77\end{array}$ & $\begin{array}{l}\mathrm{r}=0.14 \\
p=0.52\end{array}$ \\
\hline
\end{tabular}

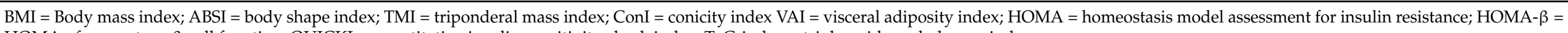
HOMA of percentage $\beta$-cell function; QUICKI = quantitative insulin sensitivity check index; TyG-index = triglyceride and glucose index. 
Other correlations between adiposity indexes and IR parameters varied according to sex. In particular, BMI was correlated $(p<0.001)$ with all indirect IR parameters except for the TyG-index in females. Fat mass and TMI correlated with all IR parameters only in females; BMI z-score correlated with IR markers except for HOMA- $\beta$ in males $(p>0.05)$; $\mathrm{WC} / \mathrm{H}$ was not correlated with IR indexes except with HOMA- $\beta$ in both sexes (males $p=0.01$; females $p=0.04$ ); Free fat mass was related to HOMA-IR and QUICKI only in females $(p<0.01)$; ConI was only correlated with the TyG-index in females $(p=0.01)$. ABSI was not correlated with IR measurements.

As reported in Table 2, all significant correlations were influenced by puberty in both males and females. For BMI, BMI-z score, WC, Fat mass, free fat mass, VAI, TMI, and ConI, the correlation with IR parameters lost significance in late puberty, particularly in females, Table 2.

The differences between females and males were more evident in middle puberty, in particular for fat mass, free fat mass, VAI, TMI and Con I $(p<0.01)$.

\subsection{Correlation between IR Measurements and Cardiometabolic Parameters}

As shown in Table 3, in both sexes, triglycerides and SBP significantly correlated with all indirect IR measurements $(p<0.001)$.

Table 3. Correlation between insulin resistance measurements and biochemical parameters.

\begin{tabular}{|c|c|c|c|c|c|c|c|c|c|c|c|c|}
\hline \multirow{3}{*}{ Biochemical Parameters } & \multicolumn{12}{|c|}{ Indirect Insulin Resistance Measurements } \\
\hline & \multicolumn{3}{|c|}{ HOMA-IR } & \multicolumn{3}{|c|}{ TyG-Index } & \multicolumn{3}{|c|}{ HOMA- $\beta$} & \multicolumn{3}{|c|}{ QUICK Index } \\
\hline & All & Females & Males & All & Females & Males & All & Females & Males & All & Females & Males \\
\hline Total cholesterol & $\begin{array}{l}\mathrm{r}=0.03 \\
p=0.37\end{array}$ & $\begin{array}{l}\mathrm{r}=0.03 \\
p=0.49\end{array}$ & $\begin{array}{l}\mathrm{r}=0.04 \\
p=0.42\end{array}$ & $\begin{array}{l}r=0.28 \\
p<0.001\end{array}$ & $\begin{array}{l}\mathrm{r}=0.35 \\
p<0.001\end{array}$ & $\begin{array}{l}r=0.23 \\
p<0.001\end{array}$ & $\begin{array}{l}\mathrm{r}=0.07 \\
p=0.06\end{array}$ & $\begin{array}{l}\mathrm{r}=0.07 \\
p=0.17\end{array}$ & $\begin{aligned} \mathrm{r} & =0.1 \\
p & =0.06\end{aligned}$ & $\begin{array}{l}\mathrm{r}=0.01 \\
p=0.71\end{array}$ & $\begin{array}{l}\mathrm{r}=0.05 \\
p=0.37\end{array}$ & $\begin{array}{c}\mathrm{r}=-0.006 \\
p=0.91\end{array}$ \\
\hline Tanner 1 & $\begin{array}{c}\mathrm{r}=-0.03 \\
p=0.63\end{array}$ & $\begin{array}{c}\mathrm{r}=-0.03 \\
p=0.75\end{array}$ & $\begin{array}{c}\mathrm{r}=-0.05 \\
p=0.68\end{array}$ & $\begin{array}{l}\mathrm{r}=0.22 \\
p<0.01\end{array}$ & $\begin{array}{l}\mathrm{r}=0.33 \\
p<0.01\end{array}$ & $\begin{array}{l}\mathrm{r}=0.06 \\
p=0.65\end{array}$ & $\begin{array}{l}\mathrm{r}=0.04 \\
p=0.57\end{array}$ & $\begin{array}{c}\mathrm{r}=-0.003 \\
p=0.97\end{array}$ & $\begin{array}{l}\mathrm{r}=0.17 \\
p=0.19\end{array}$ & $\begin{array}{c}\mathrm{r}=-0.05 \\
p=0.55\end{array}$ & $\begin{array}{c}\mathrm{r}=-0.02 \\
p=0.84\end{array}$ & $\begin{array}{c}\mathrm{r}=-0.11 \\
p=0.38\end{array}$ \\
\hline Tanner 2-3 & $\begin{array}{l}\mathrm{r}=0.04 \\
p=0.43\end{array}$ & $\begin{array}{l}\mathrm{r}=0.16 \\
p=0.03\end{array}$ & $\begin{array}{c}\mathrm{r}=-0.04 \\
p=0.53\end{array}$ & $\begin{aligned} r & =0.30 \\
p & <0.001\end{aligned}$ & $\begin{aligned} \mathrm{r} & =0.37 \\
p & <0.001\end{aligned}$ & $\begin{array}{c}\mathrm{r}=0.26 \\
p<0.001\end{array}$ & $\begin{array}{l}\mathrm{r}=0.05 \\
p=0.29\end{array}$ & $\begin{array}{l}\mathrm{r}=0.14 \\
p=0.06\end{array}$ & $\begin{array}{l}\mathrm{r}=0.02 \\
p=0.72\end{array}$ & $\begin{array}{l}\mathrm{r}=0.02 \\
p=0.59\end{array}$ & $\begin{array}{l}\mathrm{r}=0.17 \\
p=0.02\end{array}$ & $\begin{array}{c}\mathrm{r}=-0.05 \\
p=0.43\end{array}$ \\
\hline Tanner 4-5 & $\begin{array}{l}\mathrm{r}=0.10 \\
p=0.43\end{array}$ & $\begin{array}{c}\mathrm{r}=-0.20 \\
p=0.20\end{array}$ & $\begin{array}{l}\mathrm{r}=0.47 \\
p=0.02\end{array}$ & $\begin{array}{l}\mathrm{r}=0.32 \\
p<0.01\end{array}$ & $\begin{array}{l}\mathrm{r}=0.27 \\
p=0.08\end{array}$ & $\begin{array}{l}\mathrm{r}=0.26 \\
p=0.23\end{array}$ & $\begin{array}{l}\mathrm{r}=0.29 \\
p=0.02\end{array}$ & $\begin{array}{c}\mathrm{r}=-0.08 \\
p=0.58\end{array}$ & $\begin{aligned} \mathrm{r} & =0.53 \\
p & =<0.01\end{aligned}$ & $\begin{array}{l}\mathrm{r}=0.07 \\
p=0.57\end{array}$ & $\begin{array}{c}\mathrm{r}=-0.11 \\
p=0.49\end{array}$ & $\begin{array}{l}\mathrm{r}=0.34 \\
p=0.11\end{array}$ \\
\hline HDL-cholesterol & $\begin{array}{l}\mathrm{r}=-0.16 \\
p<0.001\end{array}$ & $\begin{array}{c}\mathrm{r}=-0.09 \\
p=0.08\end{array}$ & $\begin{array}{l}\mathrm{r}=-0.25 \\
p<0.001\end{array}$ & $\begin{array}{l}\mathrm{r}=-0.30 \\
p<0.001\end{array}$ & $\begin{array}{l}\mathrm{r}=-0.26 \\
p<0.001\end{array}$ & $\begin{array}{l}\mathrm{r}=-0.35 \\
p<0.001\end{array}$ & $\begin{array}{l}\mathrm{r}=-0.21 \\
p<0.001\end{array}$ & $\begin{array}{l}\mathrm{r}=-0.18 \\
p=0.001\end{array}$ & $\begin{array}{l}\mathrm{r}=-0.28 \\
p<0.001\end{array}$ & $\begin{array}{l}\mathrm{r}=-0.19 \\
p<0.001\end{array}$ & $\begin{array}{c}\mathrm{r}=-0.12 \\
p=0.03\end{array}$ & $\begin{array}{l}\mathrm{r}=-0.29 \\
p<0.001\end{array}$ \\
\hline Tanner 1 & $\begin{aligned} \mathrm{r}=-0.13 \\
p=0.09\end{aligned}$ & $\begin{array}{c}\mathrm{r}=-0.14 \\
p=0.18\end{array}$ & $\begin{array}{c}\mathrm{r}=-0.11 \\
p=0.39\end{array}$ & $\begin{array}{l}\mathrm{r}=-0.37 \\
p<0.001\end{array}$ & $\begin{array}{l}\mathrm{r}=-0.36 \\
p<0.001\end{array}$ & $\begin{array}{c}\mathrm{r}=-0.38 \\
p<0.01\end{array}$ & $\begin{aligned} \mathrm{r} & =-0.07 \\
p & =0.36\end{aligned}$ & $\begin{array}{c}\mathrm{r}=-0.11 \\
p=0.31\end{array}$ & $\begin{array}{l}\mathrm{r}=0.01 \\
p=0.91\end{array}$ & $\begin{array}{c}\mathrm{r}=-0.14 \\
p=0.08\end{array}$ & $\begin{array}{c}\mathrm{r}=-0.14 \\
p=0.19\end{array}$ & $\begin{array}{c}\mathrm{r}=-0.14 \\
p=0.27\end{array}$ \\
\hline Tanner 2-3 & $\begin{array}{l}\mathrm{r}=-0.19 \\
p<0.001\end{array}$ & $\begin{aligned} \mathrm{r}= & =-0.10 \\
p & =0.18\end{aligned}$ & $\begin{array}{l}\mathrm{r}=-0.33 \\
p<0.001\end{array}$ & $\begin{array}{l}\mathrm{r}=-0.27 \\
p<0.001\end{array}$ & $\begin{aligned} \mathrm{r}= & =-0.23 \\
p & <0.01\end{aligned}$ & $\begin{array}{l}\mathrm{r}=-0.31 \\
p<0.001\end{array}$ & $\begin{array}{l}\mathrm{r}=-0.25 \\
p<0.001\end{array}$ & $\begin{aligned} \mathrm{r}= & =-0.21 \\
p & <0.01\end{aligned}$ & $\begin{array}{l}\mathrm{r}=-0.33 \\
p<0.001\end{array}$ & $\begin{array}{l}\mathrm{r}=-0.21 \\
p<0.001\end{array}$ & $\begin{aligned} \mathrm{r}=-0.10 \\
p=0.16\end{aligned}$ & $\begin{array}{l}\mathrm{r}=-0.33 \\
p<0.001\end{array}$ \\
\hline Tanner 4-5 & $\begin{array}{l}\mathrm{r}=0.06 \\
p=0.60\end{array}$ & $\begin{array}{c}\mathrm{r}=-0.07 \\
p=0.65\end{array}$ & $\begin{array}{l}\mathrm{r}=0.09 \\
p=0.67\end{array}$ & $\begin{aligned} \mathrm{r} r & =-0.34 \\
p & <0.01\end{aligned}$ & $\begin{array}{c}\mathrm{r}=-0.30 \\
p=0.06\end{array}$ & $\begin{array}{c}\mathrm{r}=-0.29 \\
p=0.18\end{array}$ & $\begin{array}{c}\mathrm{r}=-0.11 \\
p=0.35\end{array}$ & $\begin{array}{c}\mathrm{r}=-0.08 \\
p=0.59\end{array}$ & $\begin{array}{c}\mathrm{r}=-0.12 \\
p=0.57\end{array}$ & $\begin{array}{c}\mathrm{r}=-0.01 \\
p=0.89\end{array}$ & $\begin{array}{l}\mathrm{r}=0.005 \\
p=0.99\end{array}$ & $\begin{array}{c}\mathrm{r}=-0.007 \\
p=0.97\end{array}$ \\
\hline Tryglicerides & $\begin{aligned} \mathrm{r} & =0.27 \\
p & <0.001\end{aligned}$ & $\begin{aligned} \mathrm{r} & =0.23 \\
p & <0.001\end{aligned}$ & $\begin{array}{c}\mathrm{r}=0.32 \\
p<0.001\end{array}$ & $\begin{array}{c}r=0.88 \\
p<0.001\end{array}$ & $\begin{array}{l}r=0.90 \\
p<0.001\end{array}$ & $\begin{aligned} \mathrm{r} & =0.87 \\
p & <0.001\end{aligned}$ & $\begin{aligned} \mathrm{r} & =0.30 \\
p & <0.001\end{aligned}$ & $\begin{aligned} \mathrm{r} & =0.31 \\
p & <0.001\end{aligned}$ & $\begin{array}{l}r=0.31 \\
p<0.001\end{array}$ & $\begin{aligned} \mathrm{r} & =0.30 \\
p & <0.001\end{aligned}$ & $\begin{aligned} r & =0.26 \\
p & <0.001\end{aligned}$ & $\begin{aligned} \mathrm{r} & =0.33 \\
p & <0.001\end{aligned}$ \\
\hline Tanner 1 & $\begin{aligned} \mathrm{r} & =0.37 \\
p & <0.001\end{aligned}$ & $\begin{array}{c}r=0.31 \\
p<0.001\end{array}$ & $\begin{array}{l}\mathrm{r}=-0.46 \\
p<0.001\end{array}$ & $\begin{aligned} r & =0.93 \\
p & <0.001\end{aligned}$ & $\begin{aligned} \mathrm{r} & =0.94 \\
p & <0.001\end{aligned}$ & $\begin{array}{l}r=0.94 \\
p<0.001\end{array}$ & $\begin{aligned} \mathrm{r} & =0.40 \\
p & <0.001\end{aligned}$ & $\begin{array}{l}r=0.42 \\
p<0.001\end{array}$ & $\begin{array}{l}\mathrm{r}=0.20 \\
p=0.12\end{array}$ & $\begin{aligned} r & =0.38 \\
p & <0.001\end{aligned}$ & $\begin{array}{l}\mathrm{r}=0.32 \\
p<0.01\end{array}$ & $\begin{aligned} \mathrm{r} & =0.46 \\
p & <0.001\end{aligned}$ \\
\hline Tanner 2-3 & $\begin{aligned} \mathrm{r} & =0.27 \\
p & <0.001\end{aligned}$ & $\begin{aligned} r & =0.27 \\
p & <0.001\end{aligned}$ & $\begin{array}{l}\mathrm{r}=-0.30 \\
p<0.001\end{array}$ & $\begin{aligned} r & =0.88 \\
p & <0.001\end{aligned}$ & $\begin{aligned} \mathrm{r} & =0.89 \\
p & <0.001\end{aligned}$ & $\begin{aligned} r & =0.88 \\
p & <0.001\end{aligned}$ & $\begin{array}{l}\mathrm{r}=0.27 \\
p<0.001\end{array}$ & $\begin{aligned} r & =0.27 \\
p & <0.001\end{aligned}$ & $\begin{aligned} \mathrm{r} & =0.29 \\
p & <0.001\end{aligned}$ & $\begin{aligned} r & =0.28 \\
p & <0.001\end{aligned}$ & $\begin{aligned} r & =0.27 \\
p & <0.001\end{aligned}$ & $\begin{aligned} \mathrm{r} & =0.29 \\
p & <0.001\end{aligned}$ \\
\hline Tanner 4-5 & $\begin{array}{l}\mathrm{r}=0.11 \\
p=0.38\end{array}$ & $\begin{aligned} \mathrm{r}= & =-0.09 \\
p & =0.55\end{aligned}$ & $\begin{array}{l}\mathrm{r}=0.25 \\
p=0.25\end{array}$ & $\begin{array}{c}r=0.94 \\
p<0.001\end{array}$ & $\begin{aligned} \mathrm{r} & =0.91 \\
p & <0.001\end{aligned}$ & $\begin{array}{c}r=0.95 \\
p<0.001\end{array}$ & $\begin{array}{l}\mathrm{r}=0.16 \\
p=0.19\end{array}$ & $\begin{array}{l}\mathrm{r}=0.19 \\
p=0.22\end{array}$ & $\begin{array}{l}\mathrm{r}=0.09 \\
p=0.68\end{array}$ & $\begin{array}{l}\mathrm{r}=0.13 \\
p=0.29\end{array}$ & $\begin{array}{l}\mathrm{r}=0.04 \\
p=0.80\end{array}$ & $\begin{array}{l}\mathrm{r}=0.20 \\
p=0.37\end{array}$ \\
\hline Systolic Blood Pressure & $\begin{array}{c}r=0.34 \\
p<0.001\end{array}$ & $\begin{array}{c}\mathrm{r}=0.42 \\
p<0.001\end{array}$ & $\begin{aligned} \mathrm{r} & =0.27 \\
p & <0.001\end{aligned}$ & $\begin{array}{c}r=0.21 \\
p<0.001\end{array}$ & $\begin{array}{c}r=0.22 \\
p<0.001\end{array}$ & $\begin{array}{c}r=0.21 \\
p<0.001\end{array}$ & $\begin{array}{l}\mathrm{r}=0.25 \\
p<0.001\end{array}$ & $\begin{aligned} r & =0.40 \\
p & <0.001\end{aligned}$ & $\begin{array}{l}\mathrm{r}=0.12 \\
p=0.04\end{array}$ & $\begin{array}{c}r=0.33 \\
p<0.001\end{array}$ & $\begin{array}{c}\mathrm{r}=0.47 \\
p<0.001\end{array}$ & $\begin{array}{c}\mathrm{r}=0.23 \\
p<0.001\end{array}$ \\
\hline Tanner 1 & $\begin{array}{l}\mathrm{r}=-0.37 \\
p<0.001\end{array}$ & $\begin{array}{c}\mathrm{r}=0.44 \\
p<0.001\end{array}$ & $\begin{array}{l}\mathrm{r}=0.24 \\
p=0.08\end{array}$ & $\begin{array}{l}\mathrm{r}=0.09 \\
p=0.27\end{array}$ & $\begin{array}{l}\mathrm{r}=0.19 \\
p=0.07\end{array}$ & $\begin{array}{c}\mathrm{r}=-0.06 \\
p=0.66\end{array}$ & $\begin{aligned} & \mathrm{r}=0.28 \\
& p<0.001\end{aligned}$ & $\begin{array}{l}\mathrm{r}=0.35 \\
p<0.01\end{array}$ & $\begin{array}{l}\mathrm{r}=0.13 \\
p=0.32\end{array}$ & $\begin{aligned} \mathrm{r} & =0.40 \\
p & <0.001\end{aligned}$ & $\begin{aligned} \mathrm{r} & =0.47 \\
p & <0.001\end{aligned}$ & $\begin{array}{l}\mathrm{r}=0.27 \\
p=0.04\end{array}$ \\
\hline Tanner 2-3 & $\begin{aligned} \mathrm{r} & =0.25 \\
p & <0.001\end{aligned}$ & $\begin{aligned} \mathrm{r} & =0.44 \\
p & <0.001\end{aligned}$ & $\begin{array}{l}\mathrm{r}=0.13 \\
p=0.06\end{array}$ & $\begin{aligned} r & =0.18 \\
p & <0.001\end{aligned}$ & $\begin{array}{l}\mathrm{r}=0.22 \\
p<0.01\end{array}$ & $\begin{array}{l}\mathrm{r}=0.16 \\
p=0.02\end{array}$ & $\begin{array}{l}\mathrm{r}=0.17 \\
p<0.01\end{array}$ & $\begin{aligned} \mathrm{r} & =0.40 \\
p & <0.001\end{aligned}$ & $\begin{array}{l}\mathrm{r}=0.02 \\
p=0.72\end{array}$ & $\begin{array}{c}\mathrm{r}=0.23 \\
p<0.001\end{array}$ & $\begin{aligned} \mathrm{r} & =0.44 \\
p & <0.001\end{aligned}$ & $\begin{array}{l}\mathrm{r}=0.11 \\
p=0.13\end{array}$ \\
\hline Tanner 4-5 & $\begin{aligned} \mathrm{r} & =0.38 \\
p & =0.001\end{aligned}$ & $\begin{array}{l}\mathrm{r}=0.12 \\
p=0.44\end{array}$ & $\begin{aligned} \mathrm{r} & =0.68 \\
p & <0.001\end{aligned}$ & $\begin{array}{l}\mathrm{r}=0.39 \\
p<0.01\end{array}$ & $\begin{array}{l}\mathrm{r}=0.27 \\
p=0.08\end{array}$ & $\begin{array}{l}\mathrm{r}=0.44 \\
p=0.03\end{array}$ & $\begin{array}{l}\mathrm{r}=0.11 \\
p=0.36\end{array}$ & $\begin{array}{l}\mathrm{r}=0.10 \\
p=0.50\end{array}$ & $\begin{array}{l}\mathrm{r}=0.09 \\
p=0.66\end{array}$ & $\begin{array}{l}\mathrm{r}=0.30 \\
p=0.01\end{array}$ & $\begin{array}{l}\mathrm{r}=0.19 \\
p=0.21\end{array}$ & $\begin{array}{l}\mathrm{r}=0.43 \\
p=0.04\end{array}$ \\
\hline Diastolic blood pressure & $\begin{array}{c}\mathrm{r}=0.22 \\
p<0.001\end{array}$ & $\begin{array}{c}r=0.23 \\
p<0.001\end{array}$ & $\begin{aligned} r & =0.23 \\
p & <0.001\end{aligned}$ & $\begin{aligned} \mathrm{r} & =0.007 \\
p & =0.85\end{aligned}$ & $\begin{aligned} \mathrm{r}=-0.06 \\
p=0.25\end{aligned}$ & $\begin{array}{l}\mathrm{r}=0.06 \\
p=0.26\end{array}$ & $\begin{aligned} \mathrm{r} & =0.19 \\
p & <0.001\end{aligned}$ & $\begin{array}{l}r=0.27 \\
p<0.001\end{array}$ & $\begin{array}{l}\mathrm{r}=0.13 \\
p=0.03\end{array}$ & $\begin{array}{l}r=0.17 \\
p<0.001\end{array}$ & $\begin{array}{c}\mathrm{r}=0.21 \\
p<0.001\end{array}$ & $\begin{array}{l}\mathrm{r}=0.14 \\
p=0.02\end{array}$ \\
\hline Tanner 1 & $\begin{aligned} \mathrm{r} & =0.27 \\
p & =0.001\end{aligned}$ & $\begin{array}{c}\mathrm{r}=0.39 \\
p<0.001\end{array}$ & $\begin{aligned} \mathrm{r} & =-0.05 \\
p & =0.68\end{aligned}$ & $\begin{array}{c}\mathrm{r}=-0.09 \\
p=0.27\end{array}$ & $\begin{aligned} \mathrm{r}=-0.03 \\
p=0.78\end{aligned}$ & $\begin{aligned} \mathrm{r}=-0.02 \\
p=0.08\end{aligned}$ & $\begin{array}{l}\mathrm{r}=0.17 \\
p=0.03\end{array}$ & $\begin{array}{l}\mathrm{r}=0.27 \\
p=0.01\end{array}$ & $\begin{aligned} \mathrm{r} & =-0.11 \\
p & =0.43\end{aligned}$ & $\begin{array}{c}r=0.22 \\
p<0.001\end{array}$ & $\begin{array}{l}\mathrm{r}=0.33 \\
p<0.01\end{array}$ & $\begin{aligned} \mathrm{r} r & =-0.03 \\
p & =0.78\end{aligned}$ \\
\hline Tanner 2-3 & $\begin{array}{l}\mathrm{r}=0.14 \\
p<0.01\end{array}$ & $\begin{array}{l}\mathrm{r}=0.22 \\
p<0.01\end{array}$ & $\begin{aligned} \mathrm{r} & =-0.08 \\
p & =0.26\end{aligned}$ & $\begin{aligned} \mathrm{r}= & =-0.02 \\
p & =0.71\end{aligned}$ & $\begin{array}{c}\mathrm{r}=-0.09 \\
p=0.25\end{array}$ & $\begin{array}{c}\mathrm{r}=-0.02 \\
p=0.78\end{array}$ & $\begin{array}{l}\mathrm{r}=0.14 \\
p<0.01\end{array}$ & $\begin{aligned} \mathrm{r} & =0.29 \\
p & <0.001\end{aligned}$ & $\begin{array}{l}\mathrm{r}=0.03 \\
p=0.59\end{array}$ & $\begin{array}{l}\mathrm{r}=0.08 \\
p=0.11\end{array}$ & $\begin{array}{l}\mathrm{r}=0.16 \\
p=0.04\end{array}$ & $\begin{array}{c}\mathrm{r}=-0.03 \\
p=0.63\end{array}$ \\
\hline Tanner 4-5 & $\begin{array}{l}r=0.28 \\
p=0.02\end{array}$ & $\begin{array}{c}\mathrm{r}=-0.14 \\
p=0.37\end{array}$ & $\begin{array}{c}\mathrm{r}=0.73 \\
p<0.001\end{array}$ & $\begin{array}{l}\mathrm{r}=0.13 \\
p=0.29\end{array}$ & $\begin{array}{c}\mathrm{r}=-0.004 \\
p=0.97\end{array}$ & $\begin{array}{l}\mathrm{r}=0.27 \\
p=0.21\end{array}$ & $\begin{array}{l}\mathrm{r}=0.08 \\
p=0.51\end{array}$ & $\begin{array}{c}\mathrm{r}=-0.17 \\
p=0.28\end{array}$ & $\begin{array}{l}\mathrm{r}=0.33 \\
p=0.12\end{array}$ & $\begin{array}{l}\mathrm{r}=0.09 \\
p=0.45\end{array}$ & $\begin{array}{c}\mathrm{r}=-0.22 \\
p=0.17\end{array}$ & $\begin{array}{l}\mathrm{r}=0.51 \\
p=0.01\end{array}$ \\
\hline
\end{tabular}

BMI = Body mass index; ABSI = body shape index; TMI = triponderal mass index; ConI = conicity index VAI = visceral adiposity index; HOMA = homeostasis model assessment for insulin resistance; HOMA- $\beta=$ HOMA of percentage $\beta$-cell function; QUICKI = quantitative insulin sensitivity check index; TyG-index = triglyceride and glucose index. 
Total cholesterol levels did not correlate with IR indexes, except for the TyG-index in both sexes $(p<0.001)$. DBP was not correlated with the TyG-index in females or males. HDL-cholesterol correlated with all indirect IR $(p<0.001)$ parameters, except for HOMA-IR and QUICKI in females.

Correlations between parameters and the differences between the sexes were significantly more evident in middle puberty, Table 3.

\section{Discussion}

We reported sex-specific differences in the relationship between IR and adiposity indexes, such as BMI, BMI-z score, WC, fat mass, free fat, TMI, ConI, ABSI, VAI, in children and adolescents with obesity. Sex differences were also noted in the correlation between IR markers and associated cardiometabolic risk factors, including lipidic profile and blood pressure. Puberty, body-fat distribution, and body composition may play a role in the differences between the sexes.

Childhood obesity is a multisystem condition associated with several metabolic diseases [39]. IR is an early manifestation of dysmetabolism in pediatric patients, and is also considered a precursor and risk factor for other cardiovascular diseases [1-5]. IR is characterized by an inappropriate compensatory insulin secretory response, which leads to hyperinsulinemia [40].

In this study, we observed sex-specific differences in the relationship between IR and adiposity indexes in children and adolescents with obesity. Gender differences were also noted in the correlation between IR markers and associated cardiometabolic risk factors. Puberty, body-fat distribution, and body composition may play a role in these differences.

Some simple methods, including HOMA-IR, HOMA- $\beta$, QUICKI, have been validated for use in epidemiological and clinical studies to detect IR and $\beta$-cell function and predict the development of diabetes in a non-diabetic population [8]. Recently, the TyG-index has also been proposed for IR estimation [11,12].

Several factors are known to contribute to IR development in patients with obesity. Body composition, including distribution of body fat and accumulation of excess fat in certain depots is a crucial player in the development of IR to the extent that visceral fat accumulation has been shown to be a determinant of IR and cardiometabolic risk [16-32,41]. In pediatrics, pubertal changes in insulin sensitivity have been reported to contribute to IR [42].

BMI, WC, and WHtR have been widely used as indexes of obesity, because of their practical application. However, new parameters such as ABSI, VAI, TMI and the C-Index have been proposed to better evaluate body-fat distribution [16-32,41]. In our population significant differences were noted between these parameters according to sex and pubertal stage, supporting the concept of differences in adipose tissue distribution according to gender and hormonal influence [43].

Data on the correlations between new markers of adiposity and IR in pediatrics are limited [28,32]. We describe here for the first time, sex-specific differences with respect to these correlations. In both males and females, we observed that WC and VAI were closely associated with IR indexes, confirming that these parameters are valuable indicators of adipose tissue dysfunction [43] and are better predictors of impaired glucose metabolism compared to BMI and other anthropometric measurements $[44,45]$. In support of this concept, there is evidence that visceral fat is associated with adipokine production which play a key role in insulin sensitivity [44]. Additionally, the capacity of IR indexes to estimate muscle or central insulin sensitivity should be considered [8-12].

Other indexes highlighted different correlations with IR parameters according to sex, even though IR indexes were similar in males and females. In particular, some measurements, including total fat mass and TMI, were correlated with IR parameters only in females. These results are supported by the well-known sex differences in adipose tissue distribution [43]. Due to differences in the timing of pubertal development between females and males, the role of sexual hormones was also considered [42]. Moreover, different 
adipose tissue functions according to gender, such as adipokine secretion related to insulin sensitivity, cannot be excluded [43]. For example, it has been reported that developmental genes may contribute to depot- and sex-specific properties of adipose tissue [43].

In this study, ABSI was not correlated with IR indexes, showing that this parameter is a poor predictor of IR [46]. Bouchi et al. have reported that ABSI related to abdominal vs. peripheral fat ratio is positively correlated with visceral adipose depot and is supposed to be BMI-independent [47]. ABSI has also been shown to be significantly associated with cardiometabolic risk markers in the pediatric population with overweight or obesity [28]; as reported this risk may be influenced by other variables, such as hypertension [41].

As with ABSI, ConI was a poor predictor of IR considering parameters based on fasting levels of insulin and glucose. On the contrary, in females it was related to the TyG-index, supporting a role in dysmetabolism linked to hypertension and dyslipidemia rather than to IR [48].

In this study, sex-specific differences were also observed in the correlation between IR surrogates and associated cardiometabolic risk factors, such as total cholesterol, HDLcholesterol, and DBP. These data support the idea that additional genetic and lifestyle factors including dietary patterns and exercise, may also influence IR and the dysmetabolic profile $[1,5,49]$.

Western diets poor in fruit and vegetables and rich in animal-based sources of proteins affect insulin secretion and reduce IR by insufficient intake of antioxidant food compounds; negative microbiota interactions and increased metabolic acid load that have also been linked to IR and impaired glucose homeostasis [50].

Data on the impact of sex-specific differences on health are scarce in the pediatric age. The study of sex-specific differences in obesity-related complications, such as prevalence, severity of progression, and symptom presentation represent crucial information for prevention strategies in order to improve public health. Additionally, the critical importance of sex differences as modulators of drug response have been reported [51]. Thus, these differences should be considered for tailored treatments also in pediatrics.

Study limitations The study has some limitations that should be considered. First, even though the gold standard for IR determination is the euglycaemic-hyperinsulinaemic clamp technique, we used the indirect measurement of IR since the clamp is difficult to use in routine clinical practice or large epidemiologic studies, particularly in children. Secondly, we only evaluated correlations between IR and adiposity indexes; however, the role of physical activity and diet in insulin sensitivity, should be considered. Additionally, in females with adipomastia normal breast development with female Tanner classification may be difficult to evaluate. However, the Tanner scale is the gold standard in rating pubertal development in clinical practice.

Finally, sex hormone evaluations were not included in the analysis and their influence on IR cannot be excluded.

\section{Conclusions}

The relationship between IR surrogates and obesity indexes is influenced by gender in pediatrics and puberty represents the period in which these correlations were more evident. Sex-specific differences in obesity-related complications are meaningful in the detection process of pre-disease stage and in therapeutic intervention planning for children and adolescents.

Author Contributions: Conceptualization, V.C., E.V.; formal analysis, A.D.S.; investigation, L.S., S.V., F.V., C.M.; writing-original draft preparation, V.C., E.V., H.C., C.M.; writing—review and editing, V.C., E.V., H.C., C.M., G.Z.; supervision, G.Z. All authors have read and agreed to the published version of the manuscript.

Funding: This research received no external funding. 
Institutional Review Board Statement: The Institutional Review Boards of the participating Hospitals approved the study (protocol numbers 2015/ST/135 MI, P_20190030254 PV, 2020/ST/234 MI) and the study was conducted in accordance with the Helsinki Declaration of 1975, as revised in 2008.

Informed Consent Statement: All participants or their responsible guardians gave their written consent after being informed about the nature of the study.

Data Availability Statement: Data reported in this study are available upon request from the corresponding author.

Acknowledgments: The authors thank L. Kelly for English revision of the manuscript.

Conflicts of Interest: The authors have no conflict of interest to declare.

\section{References}

1. Saltiel, A.R. Insulin signaling in health and disease. J. Clin. Investig. 2021, 131. [CrossRef] [PubMed]

2. Harvey, I.; Boudreau, A.; Stephens, J.M. Adipose tissue in health and disease. Open Biol. 2020, 10, 200291. [CrossRef] [PubMed]

3. Alzaim, I.; Hammoud, S.H.; Al-Koussa, H.; Ghazi, A.; Eid, A.H.; El-Yazbi, A.F. Adipose Tissue Immunomodulation: A Novel Therapeutic Approach in Cardiovascular and Metabolic Diseases. Front. Cardiovasc. Med. 2020, 7, 602088. [CrossRef]

4. Pastore, I.; Bolla, A.M.; Montefusco, L.; Lunati, M.E.; Rossi, A.; Assi, E.; Zuccotti, G.V.; Fiorina, P. The Impact of Diabetes Mellitus on Cardiovascular Risk Onset in Children and Adolescents. Int. J. Mol. Sci. 2020, 21, 4928. [CrossRef]

5. Castorani, V.; Polidori, N.; Giannini, C.; Blasetti, A.; Chiarelli, F. Insulin resistance and type 2 diabetes in children. Ann. Pediatric Endocrinol. Metab. 2020, 25, 217-226. [CrossRef] [PubMed]

6. Muniyappa, R.; Lee, S.; Chen, H.; Quon, M.J. Current approaches for assessing insulin sensitivity and resistance in vivo: Advantages, limitations, and appropriate usage. Am. J. Physiol. Metab. 2008, 294, E15-E26. [CrossRef] [PubMed]

7. DeFronzo, R.A.; Tobin, J.D.; Andres, R. Glucose clamp technique: A method for quantifying insulin secretion and resistance. Am. J. Physiol. 1979, 237, E214-E223. [CrossRef] [PubMed]

8. Matthews, D.R.; Hosker, J.P.; Rudenski, A.S.; Naylor, B.A.; Treacher, D.F.; Turner, R.C. Homeostasis model assessment: Insulin resistance and beta-cell function from fasting plasma glucose and insulin concentrations in man. Diabetologia 1985, 28, 412-419. [CrossRef] [PubMed]

9. Chen, H.; Sullivan, G.; Yue, L.Q.; Katz, A.; Quon, M.J. QUICKI is a useful index of insulin sensitivity in subjects with hypertension. Am. J. Physiol. Endocrinol. Metab. 2003, 284, E804-E812. [CrossRef] [PubMed]

10. McAuley, K.A.; Williams, S.M.; Mann, J.I.; Walker, R.J.; Lewis-Barned, N.J.; Temple, L.A.; Duncan, A.W. Diagnosing insulin resistance in the general population. Diabetes Care 2001, 24, 460-464. [CrossRef] [PubMed]

11. Sánchez-García, A.; Rodríguez-Gutiérrez, R.; Mancillas-Adame, L.; González-Nava, V.; González-Colmenero, A.D.; Solis, R.C.; Álvarez-Villalobos, N.A.; González-González, J.G. Diagnostic Accuracy of the Triglyceride and Glucose Index for Insulin Resistance: A Systematic Review. Int. J. Endocrinol. 2020, 2020, 1-7. [CrossRef]

12. Calcaterra, V.; Montalbano, C.; De Silvestri, A.; Pelizzo, G.; Regalbuto, C.; Paganelli, V.; Albertini, R.; Cave, F.D.; Larizza, D.; Cena, H. Triglyceride Glucose Index as a Surrogate Measure of Insulin Sensitivity in a Caucasian Pediatric Population. J. Clin. Res. Pediatric Endocrinol. 2020, 15. [CrossRef]

13. Paiman, E.H.; de Mutsert, R.; Widya, R.L.; Rosendaal, F.R.; Jukema, J.W.; Lamb, H.J. The role of insulin resistance in the relation of visceral, abdominal subcutaneous and total body fat to cardiovascular function. Nutr. Metab. Cardiovasc. Dis. 2020, 30, 2230-2241. [CrossRef]

14. Farruggia, M.C.; Small, D.M. Effects of adiposity and metabolic dysfunction on cognition: A review. Physiol. Behav. 2019, 208, 112578. [CrossRef] [PubMed]

15. Bosy-Westphal, A.; Braun, W.; Geisler, C.; Norman, K.; Müller, M.J. Body composition and cardiometabolic health: The need for novel concepts. Eur. J. Clin. Nutr. 2018, 72, 638-644. [CrossRef] [PubMed]

16. World Health Organization. Waist Circumference and Waist-Hip Ratio: Report of a WHO Expert Consultation, Geneva, 8-11 December 2008; World Health Organization: Geneva, Switzerland, 2011.

17. Rodea-Montero, E.R.; Evia-Viscarra, M.L.; Apolinar-Jiménez, E. Waist-to-Height Ratio Is a Better Anthropometric Index than Waist Circumference and BMI in Predicting Metabolic Syndrome among Obese Mexican Adolescents. Int. J. Endocrinol. 2014, 2014, 1-9. [CrossRef] [PubMed]

18. Perona, J.S.; Rio-Valle, J.S.; Ramírez-Vélez, R.; Correa-Rodríguez, M.; Fernández-Aparicio, Á.; González-Jiménez, E. Waist circumference and abdominal volume index are the strongest anthropometric discriminators of metabolic syndrome in Spanish adolescents. Eur. J. Clin. Investig. 2018, 49, e13060. [CrossRef] [PubMed]

19. Sangrador, C.O.; Ochoa-Brezmes, J. Waist-to-height ratio as a risk marker for metabolic syndrome in childhood. A meta-analysis. Pediatric Obes. 2018, 13, 421-432. [CrossRef] [PubMed]

20. Ashwell, M.; Gibson, S. Waist-to-height ratio as an indicator of 'early health risk': Simpler and more predictive than using a 'matrix' based on BMI and waist circumference. BMJ Open 2016, 6, e010159. [CrossRef] [PubMed] 
21. Bertoli, S.; Leone, A.; Krakauer, N.Y.; Bedogni, G.; Vanzulli, A.; Redaelli, V.I.; De Amicis, R.; Vignati, L.; Krakauer, J.C.; Battezzati, A. Association of Body Shape Index (ABSI) with cardio-metabolic risk factors: A cross-sectional study of 6081 Caucasian adults. PLoS ONE 2017, 12, e0185013. [CrossRef] [PubMed]

22. Maffeis, C.; Banzato, C.; Talamini, G.; Obesity Study Group of the Italian Society of Pediatric Endocrinology and Diabetology. Waist-to-height ratio, a useful index to identify high metabolic risk in overweight children. J. Pediatrics 2008, 152, 207-213. [CrossRef] [PubMed]

23. Tee, J.Y.H.; Gan, W.Y.; Lim, P.Y. Comparisons of body mass index, waist circumference, waist-to-height ratio and a body shape index (ABSI) in predicting high blood pressure among Malaysian adolescents: A cross-sectional study. BMJ Open 2020, 10, e032874. [CrossRef] [PubMed]

24. Freedman, D.S.; Kahn, H.S.; Mei, Z.; Grummer-Strawn, L.M.; Dietz, W.H.; Srinivasan, S.R.; Berenson, G.S. Relation of body mass index and waist-to-height ratio to cardiovascular disease risk factors in children and adolescents: The Bogalusa Heart Study. Am. J. Clin. Nutr. 2007, 86, 33-40. [CrossRef] [PubMed]

25. Wormser, D.; Kaptoge, S.; Di Angelantonio, E.; Wood, A.M.; Pennells, L.; Thompson, A.; Sarwar, N.; Kizer, J.R.; Lawlor, D.A.; Nordestgaard, B.G.; et al. Separate and combined associations of body-mass index and abdominal adiposity with cardiovascular disease: Collaborative analysis of 58 prospective studies. Lancet 2011, 377, 1085-1095. [PubMed]

26. Wang, F.; Chen, Y.; Chang, Y.; Sun, G.; Sun, Y. New anthropometric indexes or old ones: Which perform better in estimating cardiovascular risks in Chinese adults. BMC Cardiovasc. Disord. 2018, 18, 14. [CrossRef] [PubMed]

27. Bozorgmanesh, M.; Sardarinia, M.; Hajsheikholeslami, F.; Azizi, F.; Hadaegh, F. CVD-predictive performances of "a body shape index" versus simple anthropometric measures: Tehran lipid and glucose study. Eur. J. Nutr. 2016, 55, 147-157. [CrossRef]

28. Mameli, C.; Krakauer, N.Y.; Krakauer, J.C.; Bosetti, A.; Ferrari, C.M.; Moiana, N.; Schneider, L.; Borsani, B.; Genoni, T.; Zuccotti, G. The association between a body shape index and cardiovascular risk in overweight and obese children and adolescents. PLoS ONE 2018, 13, e0190426. [CrossRef]

29. Peterson, C.M.; Su, H.; Thomas, D.M.; Heo, M.; Golnabi, A.H.; Pietrobelli, A.; Heymsfield, S.B. Tri-Ponderal Mass Index vs Body Mass Index in Estimating Body Fat During Adolescence. JAMA Pediatrics 2017, 171, 629-636. [CrossRef] [PubMed]

30. Ramírez-Vélez, R.; Correa-Bautista, J.E.; Carrillo, H.A.; González-Jiménez, E.; Schmidt-RioValle, J.; Correa-Rodríguez, M.; GarciaHermoso, A.; González-Ruíz, K. Tri-Ponderal Mass Index vs. Fat Mass/Height3 as a Screening Tool for Metabolic Syndrome Prediction in Colombian Children and Young People. Nutrients 2018, 10, 412. [CrossRef]

31. De Oliveira, R.G.; Guedes, D.P. Performance of anthropometric indicators as predictors of metabolic syndrome in Brazilian adolescents. BMC Pediatrics 2018, 18, 33. [CrossRef]

32. Leone, A.; Vizzuso, S.; Brambilla, P.; Mameli, C.; Ravella, S.; De Amicis, R.; Battezzati, A.; Zuccotti, G.; Bertoli, S.; Verduci, E. Evaluation of Different Adiposity Indices and Association with Metabolic Syndrome Risk in Obese Children: Is there a Winner? Int. J. Mol. Sci. 2020, 21, 4083. [CrossRef] [PubMed]

33. NHLBI Obesity Education Initiative Expert Panel on the Identification Evaluation and Treatment of Obesity in Adults. In Clinical Guidelines on the Identification, Evaluation, and Treatment of Overweight and Obesity in Adults; NHLBI Obesity Education Initiative Expert Panel on the Identification, Evaluation, and Treatment of Obesity in Adults (US): Bethesda, MD, USA, 1998.

34. Krakauer, N.Y.; Krakauer, J. A New Body Shape Index Predicts Mortality Hazard Independently of Body Mass Index. PLoS ONE 2012, 7, e39504. [CrossRef] [PubMed]

35. Mangla, A.G.; Dhamija, N.; Gupta, U.; Dhall, M. Anthropometric Markers as a Paradigm for Obesity Risk Assessment. J. Biosci. Med. 2020, 8, 1-16. [CrossRef]

36. Amato, M.C.; Giordano, C.; Galia, M.; Criscimanna, A.; Vitabile, S.; Midiri, M.; Galluzzo, A.; for the AlkaMeSy Study Group Visceral Adiposity Index. A reliable indicator of visceral fat function associated with cardiometabolic risk. Diabetes Care 2010, 33 , 920-922. [CrossRef] [PubMed]

37. Simental-Mendía, L.E.; Rodríguez-Morán, M.; Guerrero-Romero, F. The Product of Fasting Glucose and Triglycerides as Surrogate for Identifying Insulin Resistance in Apparently Healthy Subjects. Metab. Syndr. Relat. Disord. 2008, 6, $299-304$. [CrossRef] [PubMed]

38. Katz, A.; Nambi, S.S.; Mather, K.; Baron, A.D.; Follmann, D.A.; Sullivan, G.; Quon, M.J. Quantitative insulin sensitivity check index: A simple, accurate method for assessing insulin sensitivity in humans. Clin. Endocrinol. Metab. 2000, 85, 2402-2410. [CrossRef]

39. Calcaterra, V.; Regalbuto, C.; Porri, D.; Pelizzo, G.; Mazzon, E.; Vinci, F.; Zuccotti, G.; Fabiano, V.; Cena, H. Inflammation in Obesity-Related Complications in Children: The Protective Effect of Diet and Its Potential Role as a Therapeutic Agent. Biomolecules 2020, 10, 1324. [CrossRef]

40. Wilcox, G. Insulin and Insulin Resistance. Clin. Biochem. Rev. 2005, 26, 19-39.

41. Chung, W.; Park, C.G.; Ryu, O.-H. Association of a New Measure of Obesity with Hypertension and Health-Related Quality of Life. PLoS ONE 2016, 11, e0155399. [CrossRef] [PubMed]

42. Kelsey, M.M.; Zeitler, P.S. Insulin Resistance of Puberty. Curr. Diabetes Rep. 2016, 16, 1-6. [CrossRef] [PubMed]

43. Valencak, T.G.; Osterrieder, A.; Schulz, T.J. Sex matters: The effects of biological sex on adipose tissue biology and energy metabolism. Redox Biol. 2017, 12, 806-813. [CrossRef] [PubMed] 
44. Wei, M.; Gaskill, S.P.; Haffner, S.M.; Stern, M.P. Waist circumference as the best predictor of noninsulin dependent diabetes mellitus (NIDDM) compared to body mass index, waist/hip ratio and other anthropometric measurements in Mexican Americans-a 7-year prospective study. Obes. Res. 1997, 5, 16-23. [CrossRef]

45. Al-Daghri, N.M.; Al-Attas, O.S.; Alokail, M.; Alkharfy, K.; Wani, K.; Amer, O.E.; Haq, S.U.; Rahman, S.; Alnaami, A.M.; Livadas, S.; et al. Does visceral adiposity index signify early metabolic risk in children and adolescents?: Association with insulin resistance, adipokines, and subclinical inflammation. Pediatric Res. 2014, 75, 459-463. [CrossRef] [PubMed]

46. $\mathrm{Wu}, \mathrm{K} . ; \mathrm{He}, \mathrm{S}$;; Zheng, Y.; Chen, X. ABSI is a poor predictor of insulin resistance in Chinese adults and elderly without diabetes. Arch. Endocrinol. Metab. 2018, 62, 523-529. [CrossRef] [PubMed]

47. Bouchi, R.; Asakawa, M.; Ohara, N.; Nakano, Y.; Takeuchi, T.; Murakami, M.; Sasahara, Y.; Numasawa, M.; Minami, I.; Izumiyama, H.K.; et al. Indirect measure of visceral adiposity 'A Body Shape Index' (ABSI) is associated with arterial stiffness in patients with type 2 diabetes. BMJ Open Diabetes Res. Care 2016, 4, e000188. [CrossRef]

48. Nkwana, M.R.; Monyeki, K.D.; Lebelo, S.L. Body Roundness Index, A Body Shape Index, Conicity Index, and Their Association with Nutritional Status and Cardiovascular Risk Factors in South African Rural Young Adults. Int. J. Environ. Res. Public Health 2021, 18, 281. [CrossRef] [PubMed]

49. Cena, H.; Calder, P.C. Defining a Healthy Diet: Evidence for the Role of Contemporary Dietary Patterns in Health and Disease. Nutrients 2020, 12, 334. [CrossRef] [PubMed]

50. Della Guardia, L.; Thomas, M.A.; Cena, H. Insulin Sensitivity and Glucose Homeostasis Can Be Influenced by Metabolic Acid Load. Nutrients 2018, 10, 618. [CrossRef]

51. Özdemir, B.C.; Csajka, C.; Dotto, G.-P.; Wagner, A.D. Sex Differences in Efficacy and Toxicity of Systemic Treatments: An Undervalued Issue in the Era of Precision Oncology. J. Clin. Oncol. 2018, 36, 2680-2683. [CrossRef] [PubMed] 